Supporting Information

\title{
Iron Nanoparticle-loaded Nitrogen-Doped Carbon Nanotube/Carbon Sheet Composites Derived from MOF as Electrocatalysts for Oxygen Reduction Reaction
}

Maryam Jafari $^{1}$, Hussein Gharibi ${ }^{1} *$, Mohammad Javad Parnian ${ }^{2}$, Mokhtar Nasrollahpour $^{1}$, Mohsen Vafaee ${ }^{1}$

${ }^{1}$ Department of Chemistry, Tarbiat Modares University (TMU), Tehran, P.O. Box 14115-175, Iran.

*Corresponding author: H. Gharibi, E-mail addresses: gharibi@modares.ac.ir; h.gharibi@gmail.com

${ }^{2}$ Department of Chemistry, University of Calgary, 2500 University Dr. NW, Calgary, Alberta, T2N 4N1, Canada. 


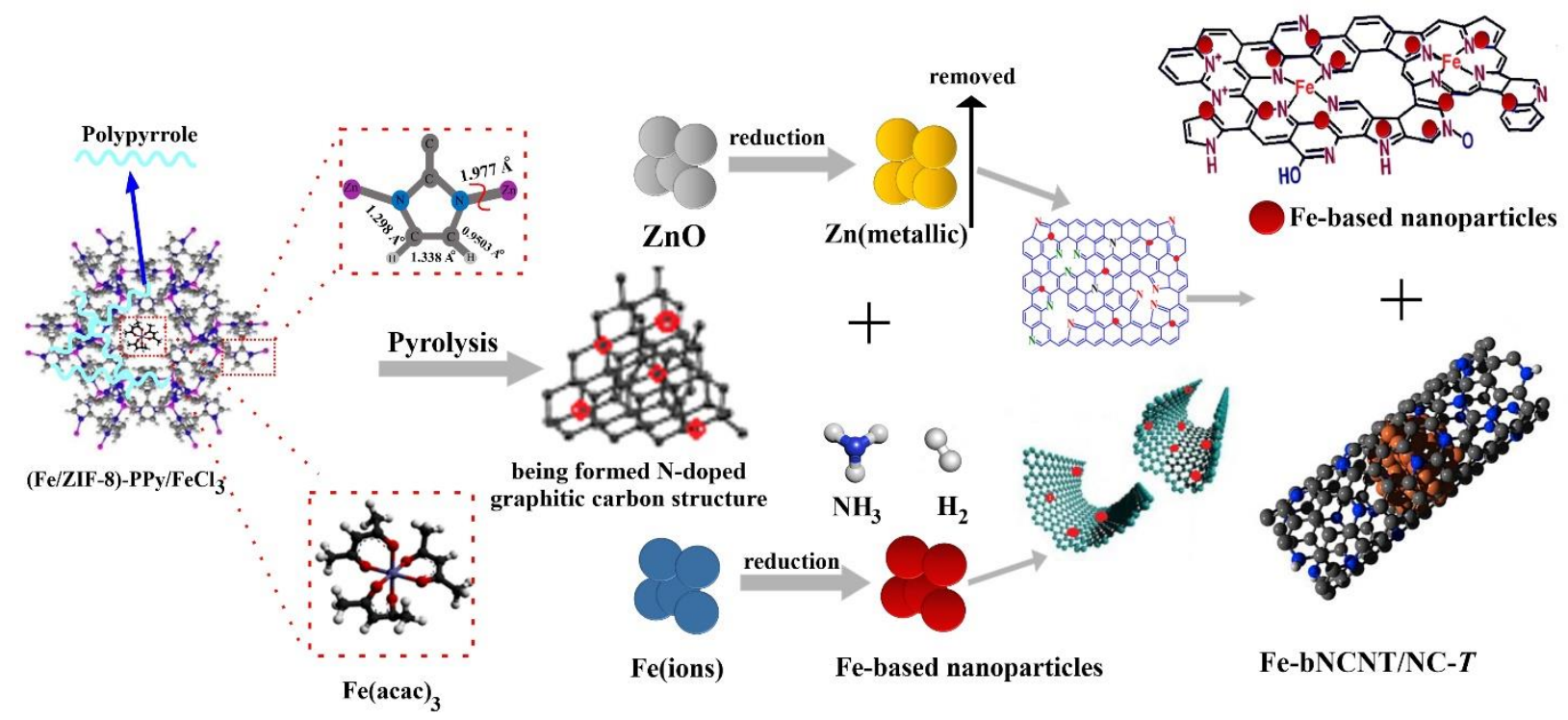

Figure S1. Schematic illustrates, in the first step, that the $\mathrm{Zn}-\mathrm{N}$ bonds have larger bond length than others, indicating preferential bond breaking. Then, producing zinc oxide, which is reduced to metallic zinc, and ultimately $\mathrm{Zn}$ evaporate from the framework. Coincidently, the generated reducing gases released through carbonization of the organic linker can reduce metal ions of $\mathrm{Fe}$ into Fe-based nanocatalysts which catalyzed the growth of $\mathrm{N}$-doped carbon sheet-like and N-doped CNTs. 

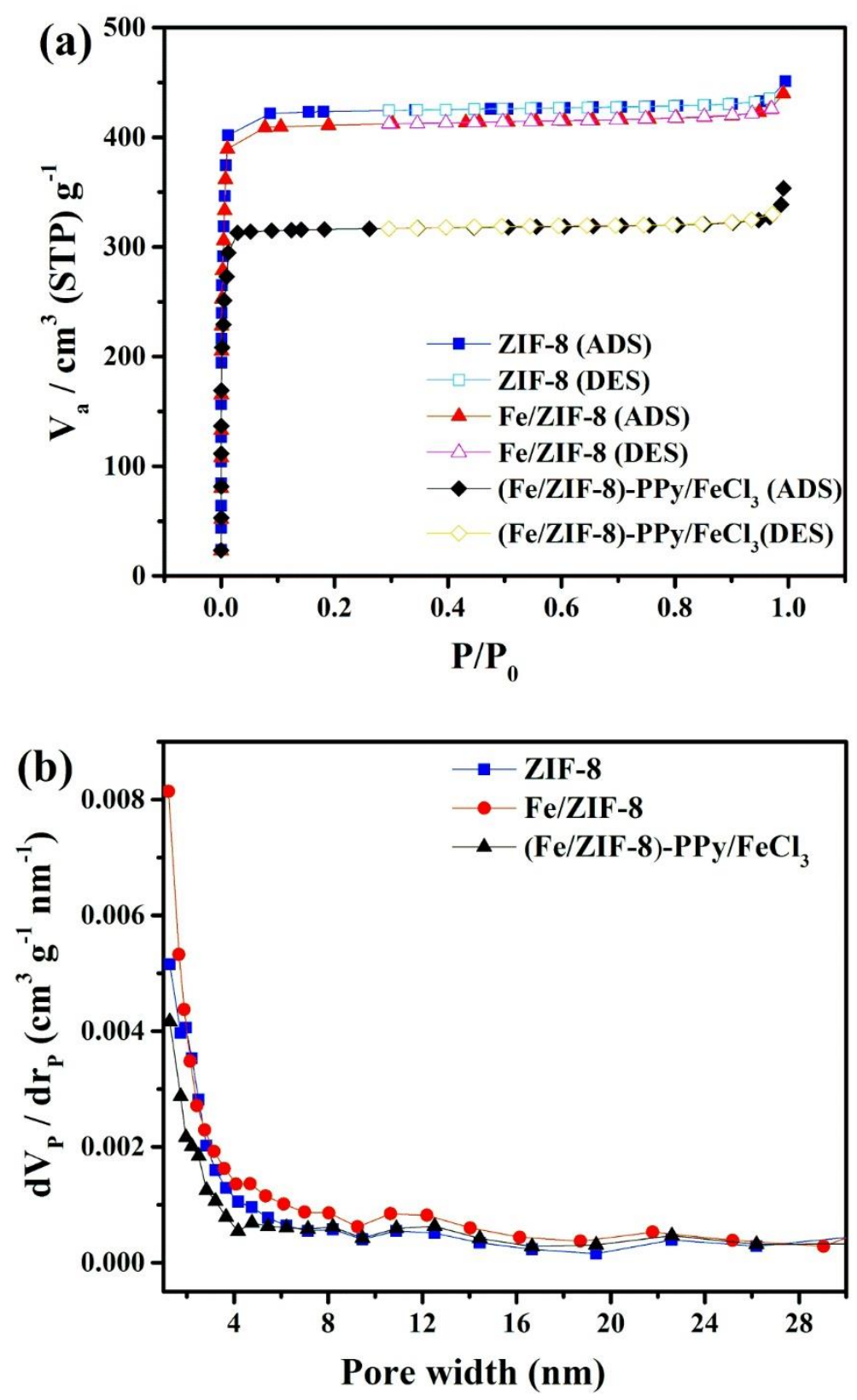

Figure S2. (a) Porous texture analysis of ZIF-8, Fe/ZIF-8, and (Fe/ZIF-8)-PPy/FeCl through $_{2}$ adsorption/desorption isotherms at $77 \mathrm{~K}$, (b) Corresponding pore size distribution curves calculated using the BJH method. 


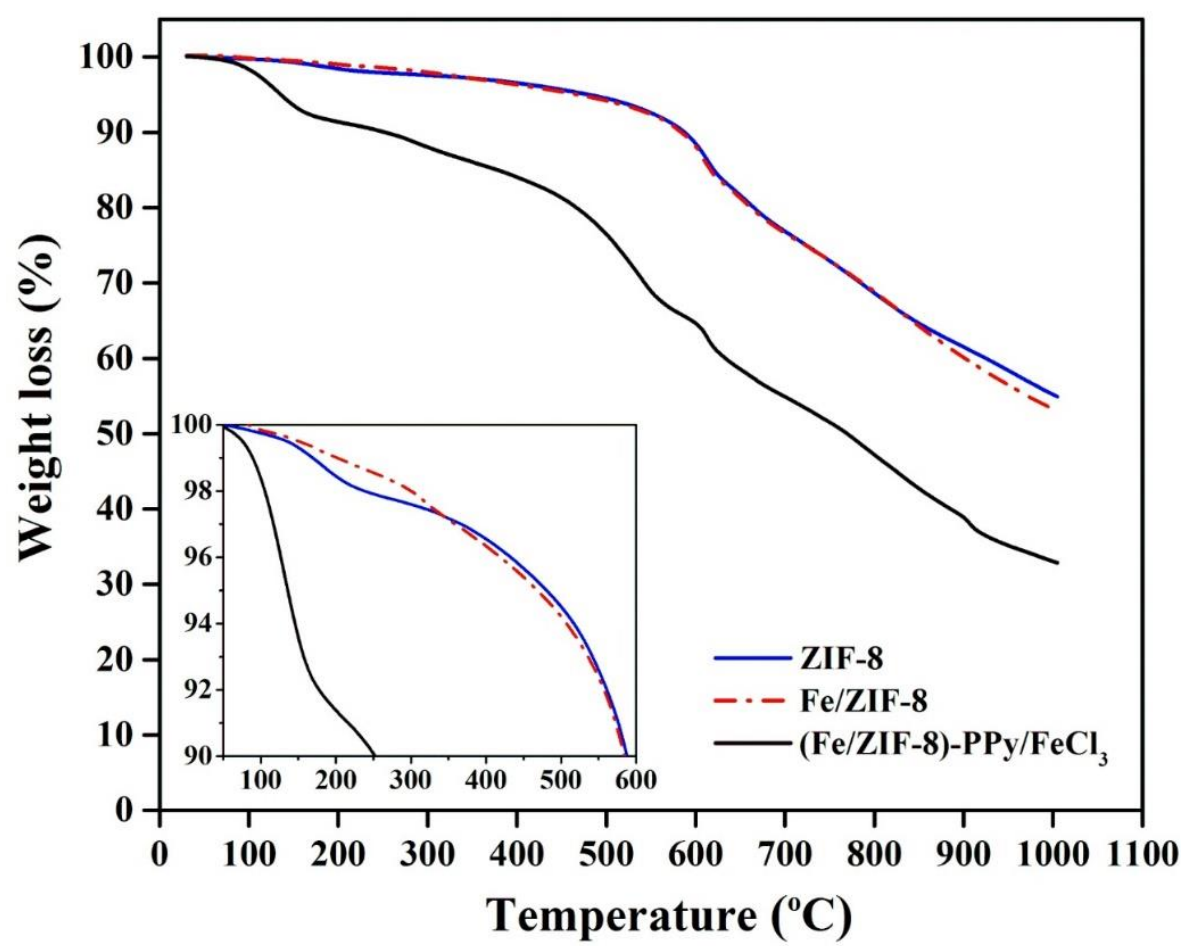

Figure S3. Thermogravimetric analysis of non-heat treated ZIF-8, Fe/ZIF-8, and (Fe/ZIF-8)$\mathrm{PPy} / \mathrm{FeCl}_{3}$ precursors. All measurements were performed under $\mathrm{N}_{2}$ atmosphere. The samples were heated at a ramp rate of $10^{\circ} \mathrm{C} \mathrm{min}^{-1}$. 

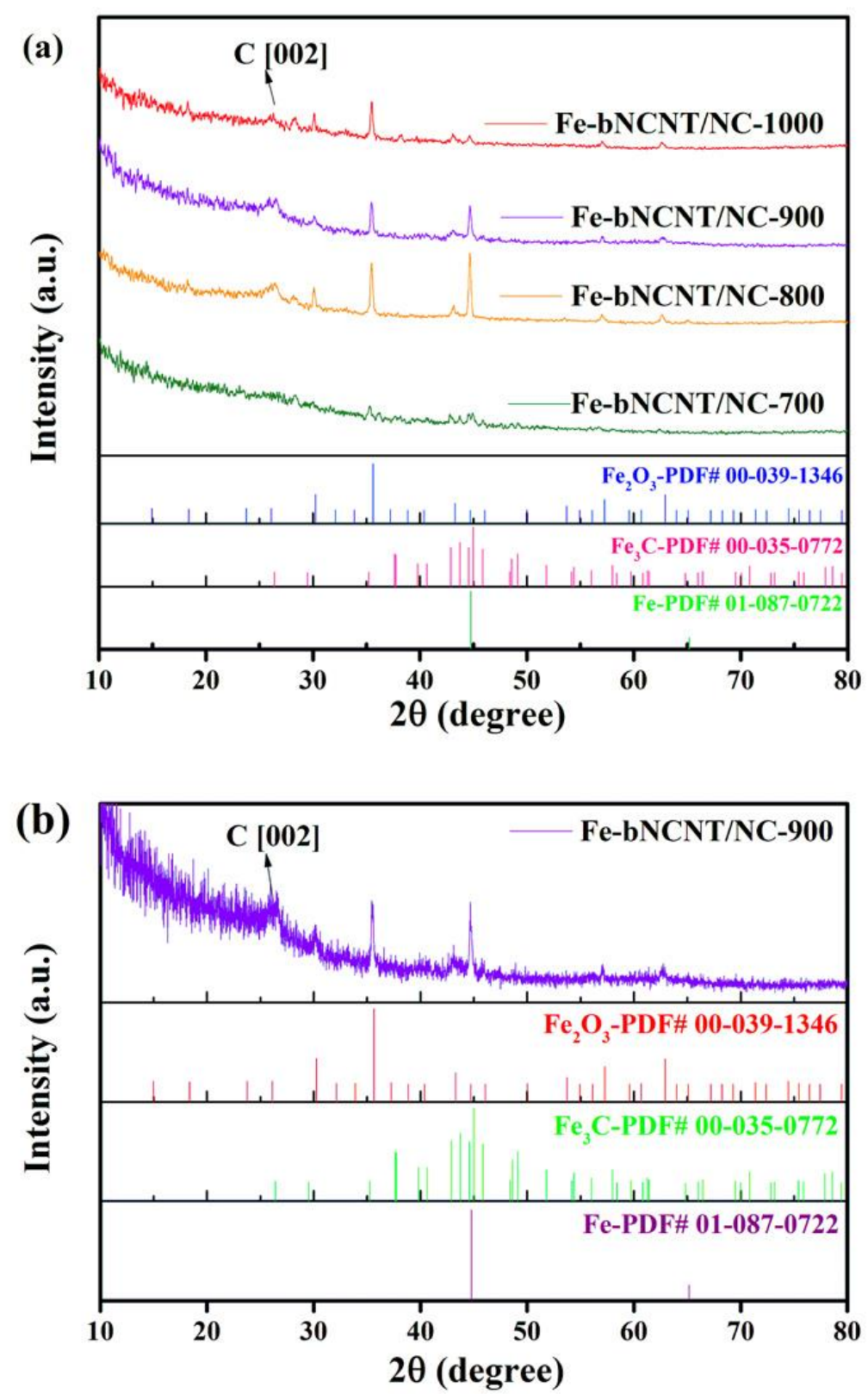

Figure S4. (a) XRD pattern of Fe-bNCNT/NC- $T$ catalysts with $\mathrm{C}, \mathrm{Fe}, \mathrm{Fe}_{3} \mathrm{C}$, and $\mathrm{Fe}_{2} \mathrm{O}_{3}$ as reference, (b) XRD pattern of Fe-bNCNT/NC-900 with $\mathrm{C}, \mathrm{Fe}, \mathrm{Fe}_{3} \mathrm{C}$, and $\mathrm{Fe}_{2} \mathrm{O}_{3}$ as reference. 

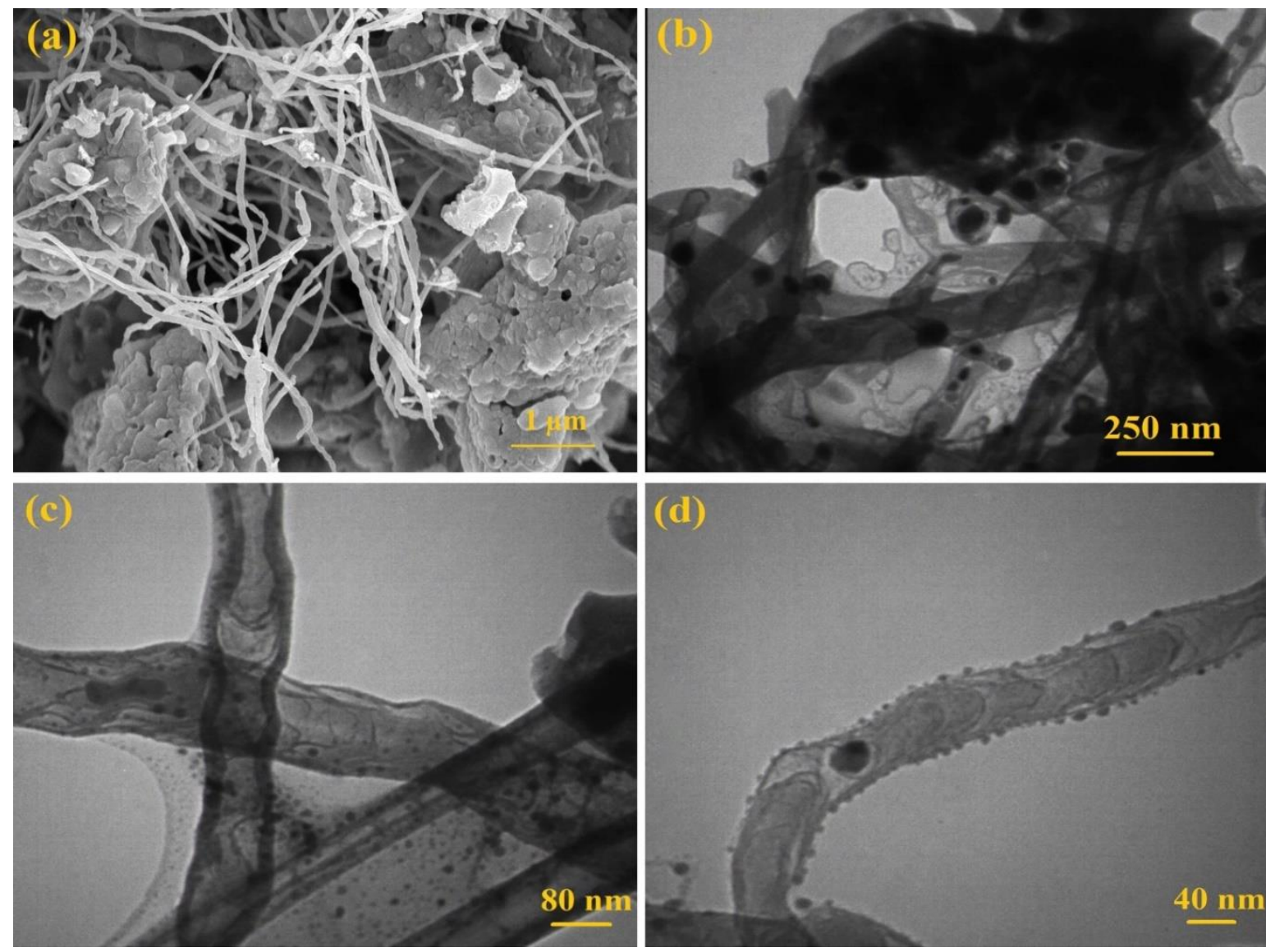

Figure S5. (a) SEM and (b-d) TEM images of Fe-bNCNT/NC-700. 

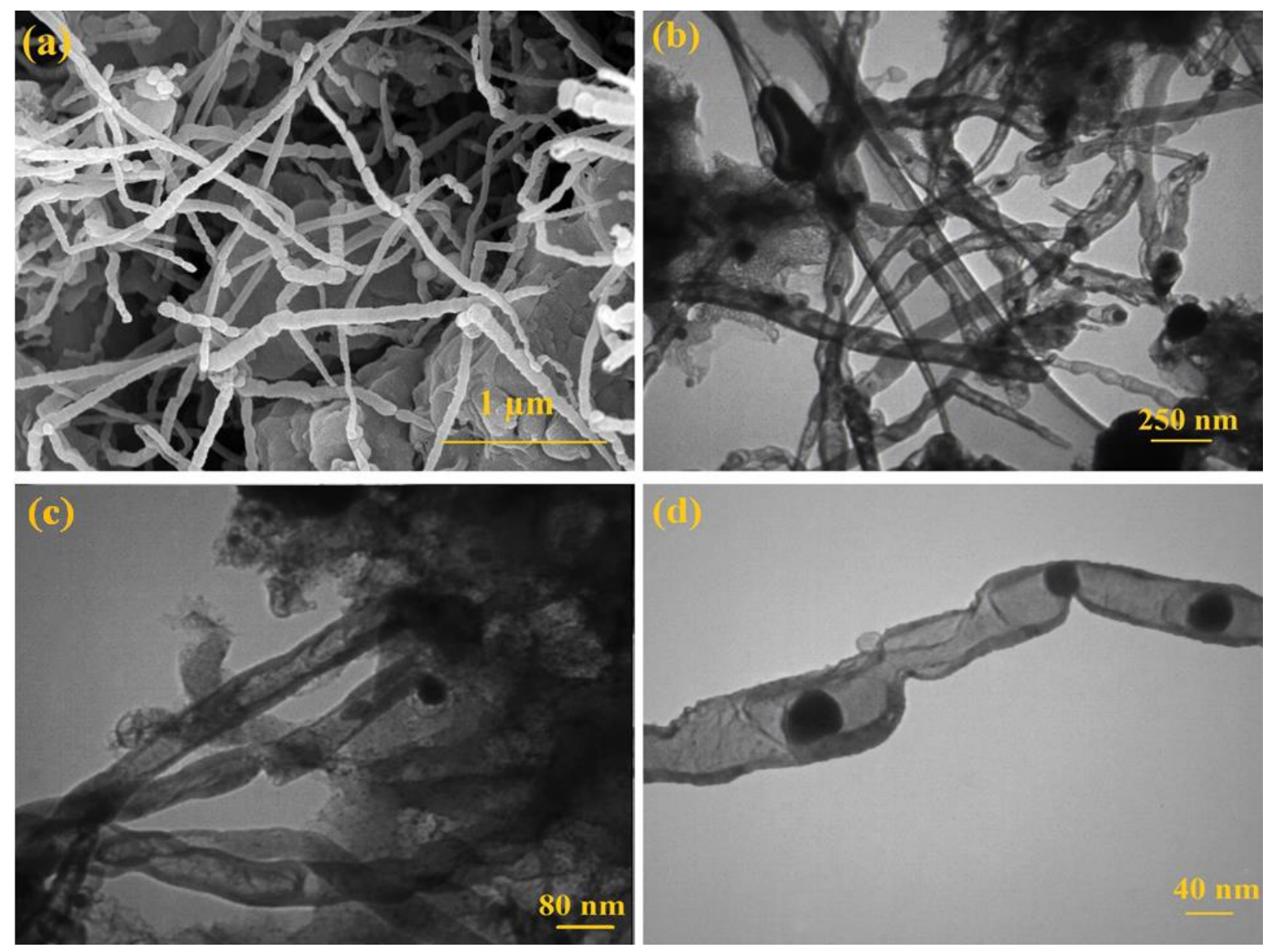

Figure S6. (a) SEM and (b-d) TEM images of Fe-bNCNT/NC-800. 

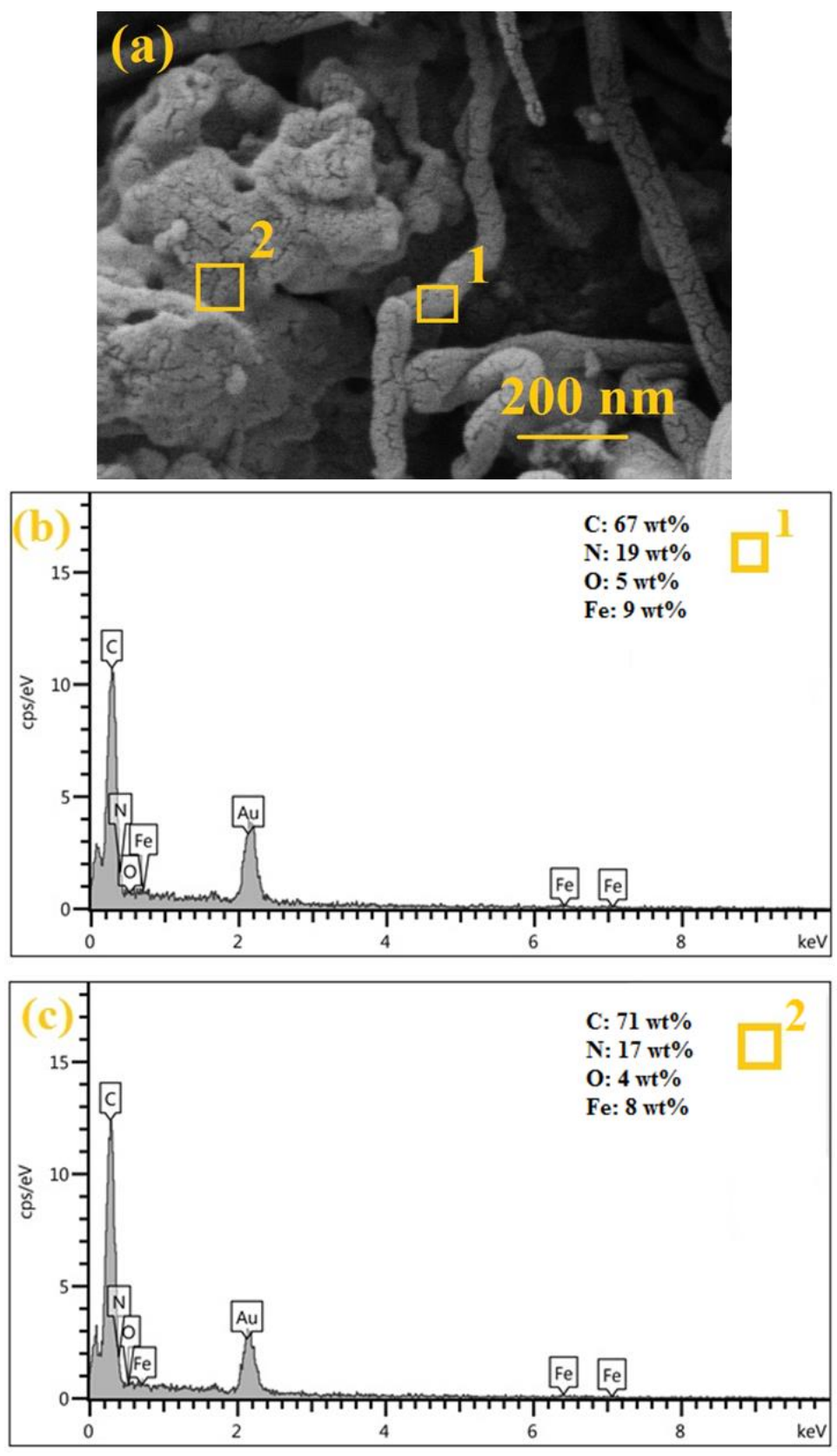

Figure S7. (a) SEM, (b-c) EDX spectra of the selected areas in (a) revealing the elemental distribution of Fe-bNCNT/NC-900. 

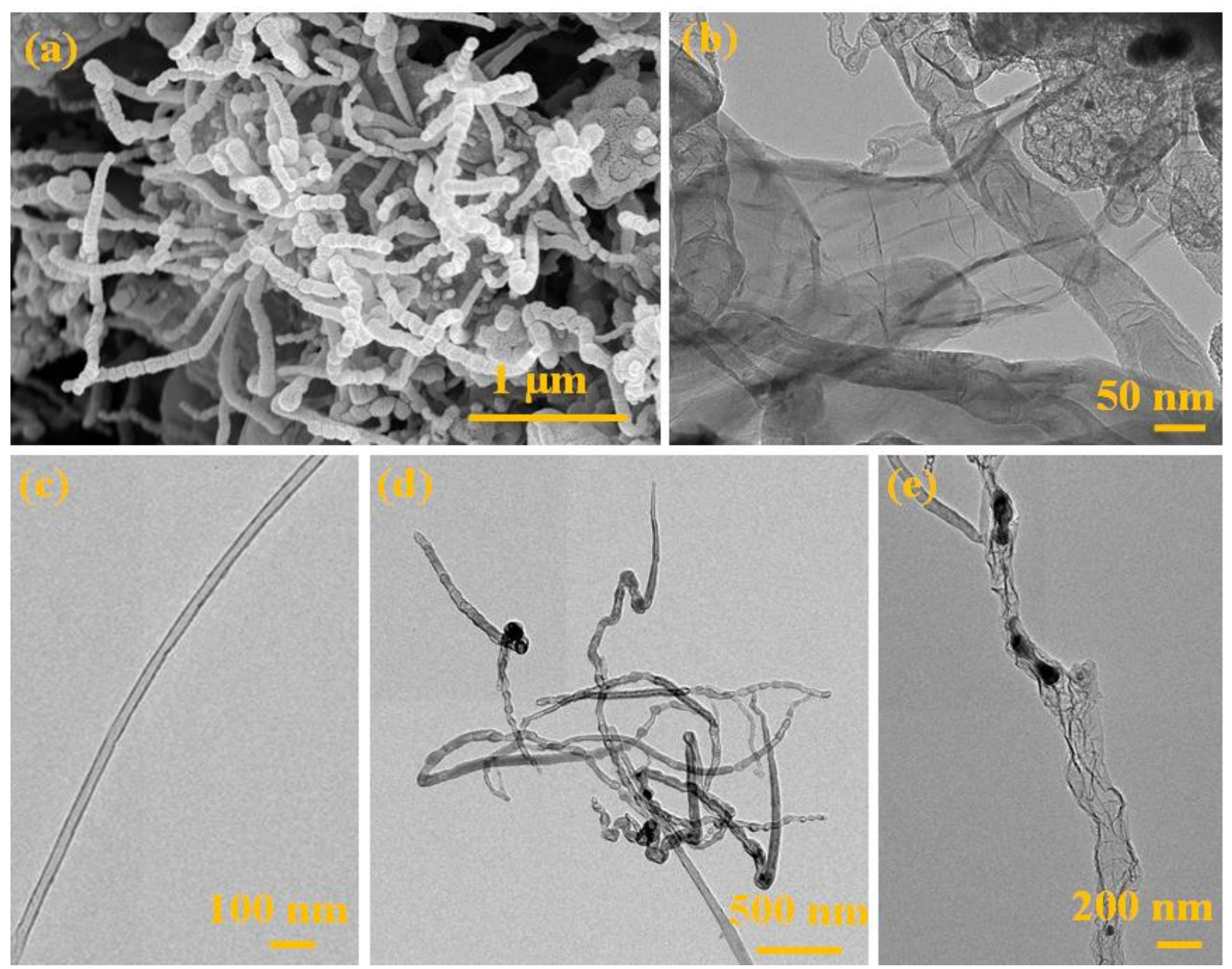

Figure S8. (a) SEM and (b-e) TEM images of Fe-bNCNT/NC-900. 

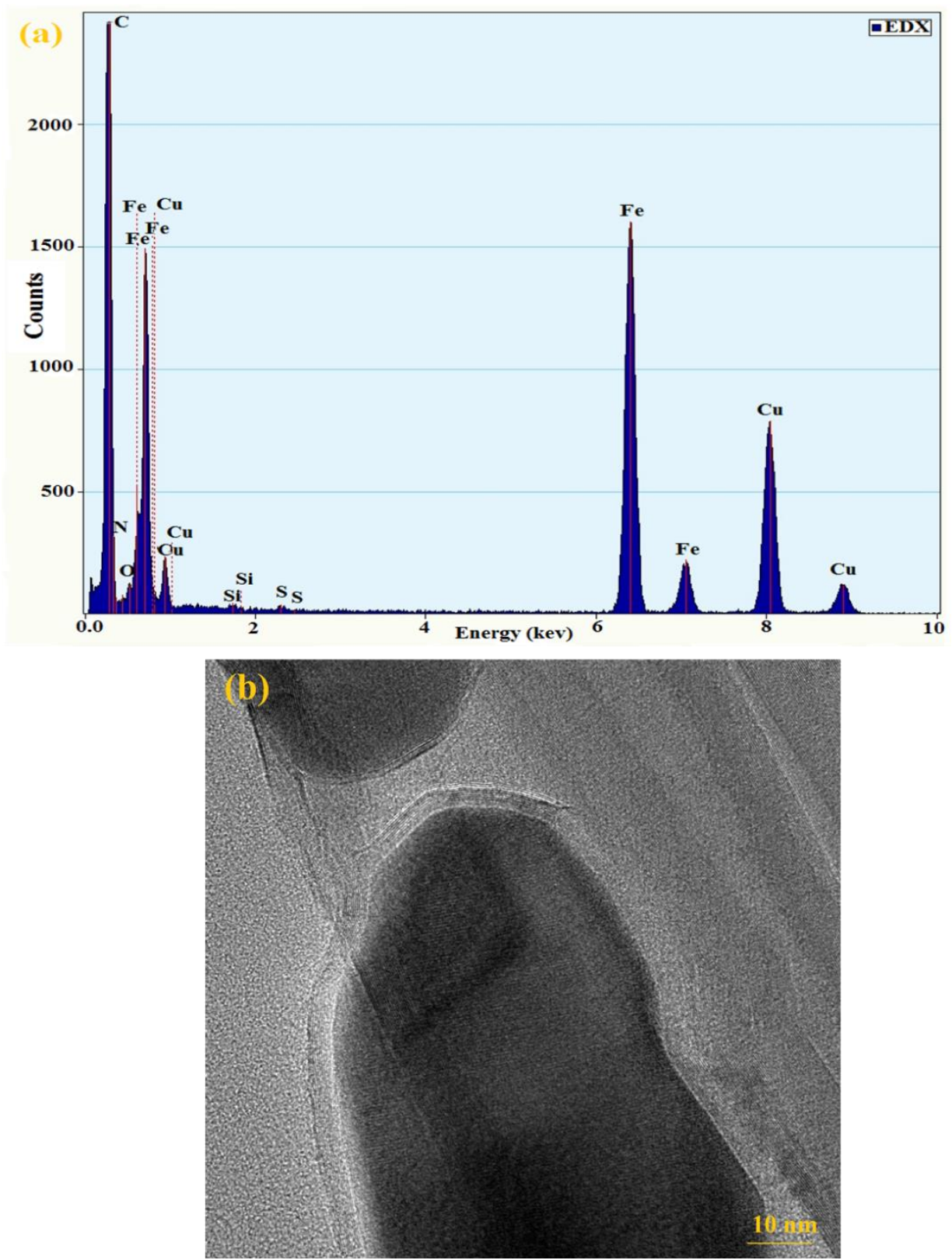

Figure S9. (a) EDX spectrum and (b) TEM image of Fe-bNCNT/NC-900. 

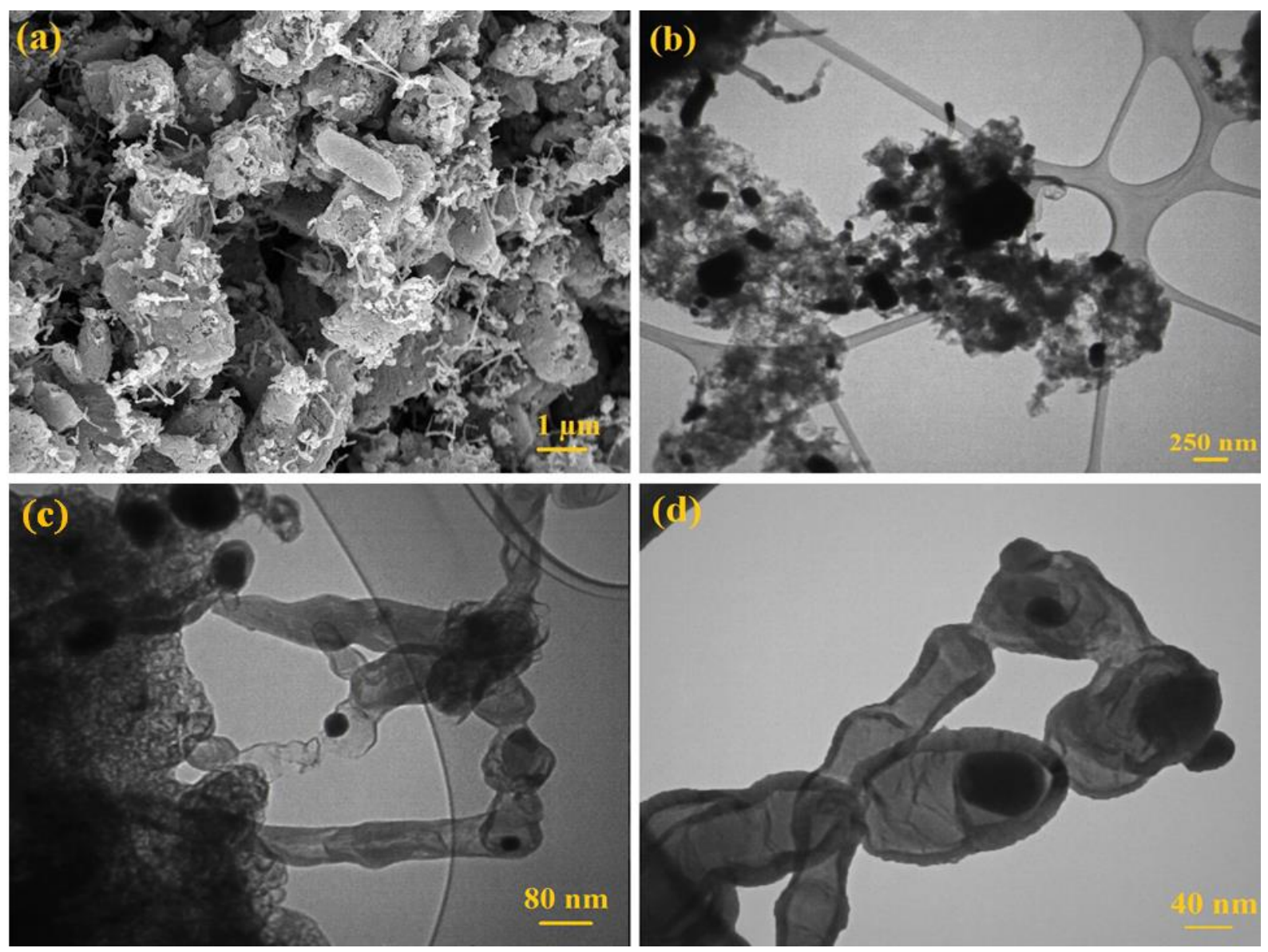

Figure S10. (a) SEM and (b-d) TEM images of Fe-bNCNT/NC-1000. 

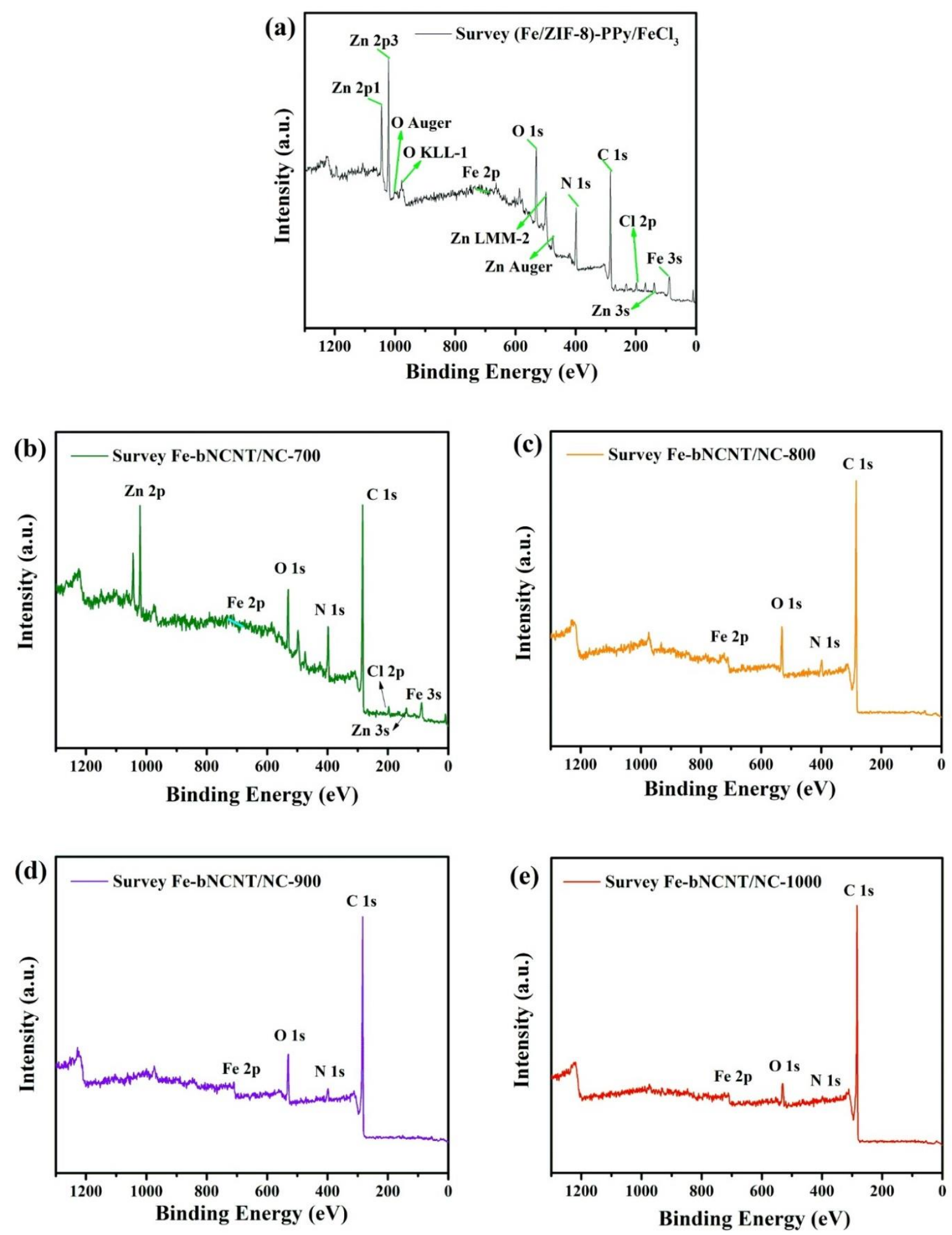

Figure S11. Survey XPS spectra of; (a) (Fe/ZIF-8)-PPy/FeCl 3 precursors, (b) Fe-bNCNT/NC-700, (c) Fe-bNCNT/NC-800, (d) Fe-bNCNT/NC-900, and (e) Fe-bNCNT/NC-1000 catalysts. 


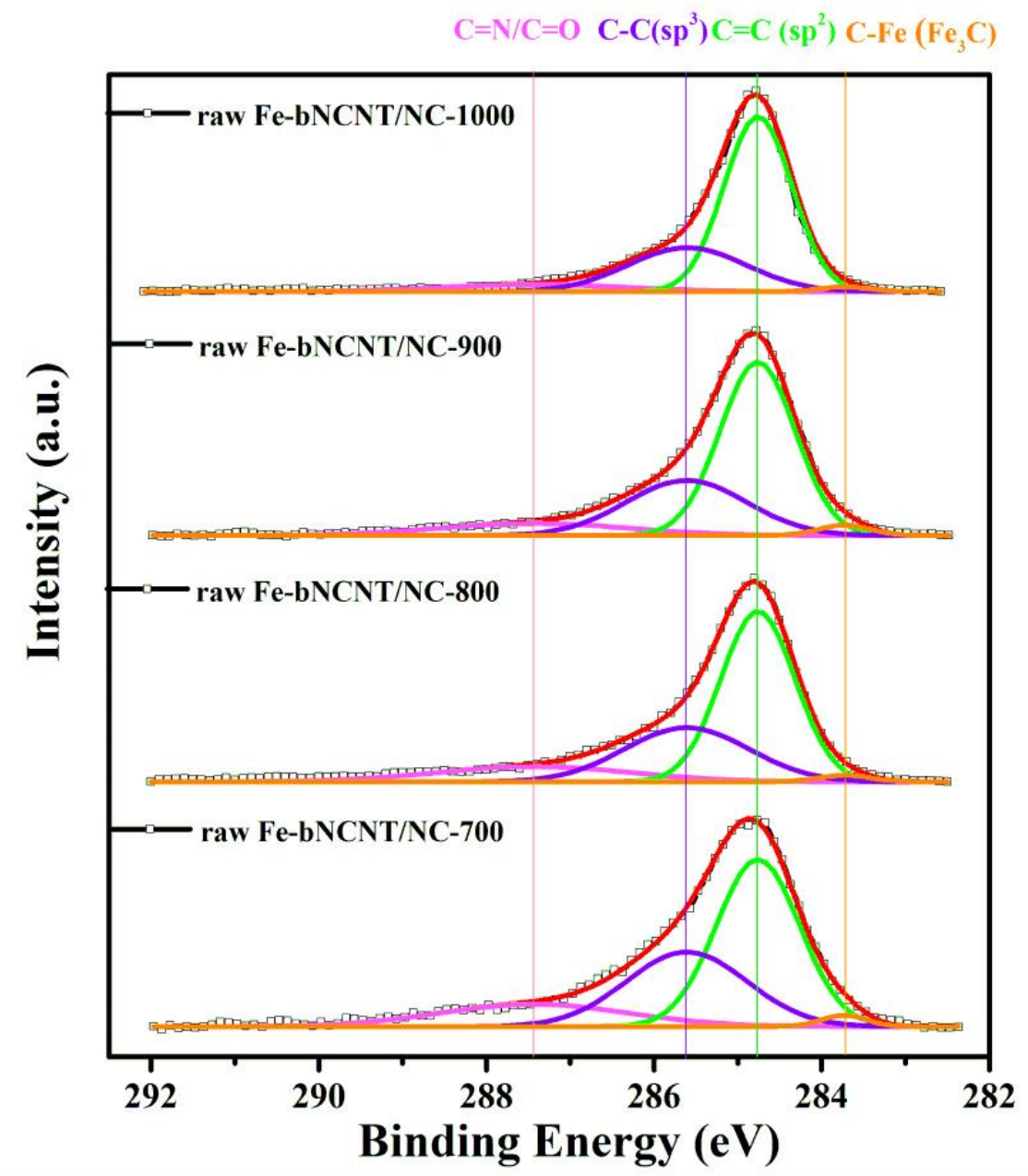

Figure S12. XPS spectra high resolution of C1s spectra of Fe-bNCNT/NC- $T$ catalysts. 


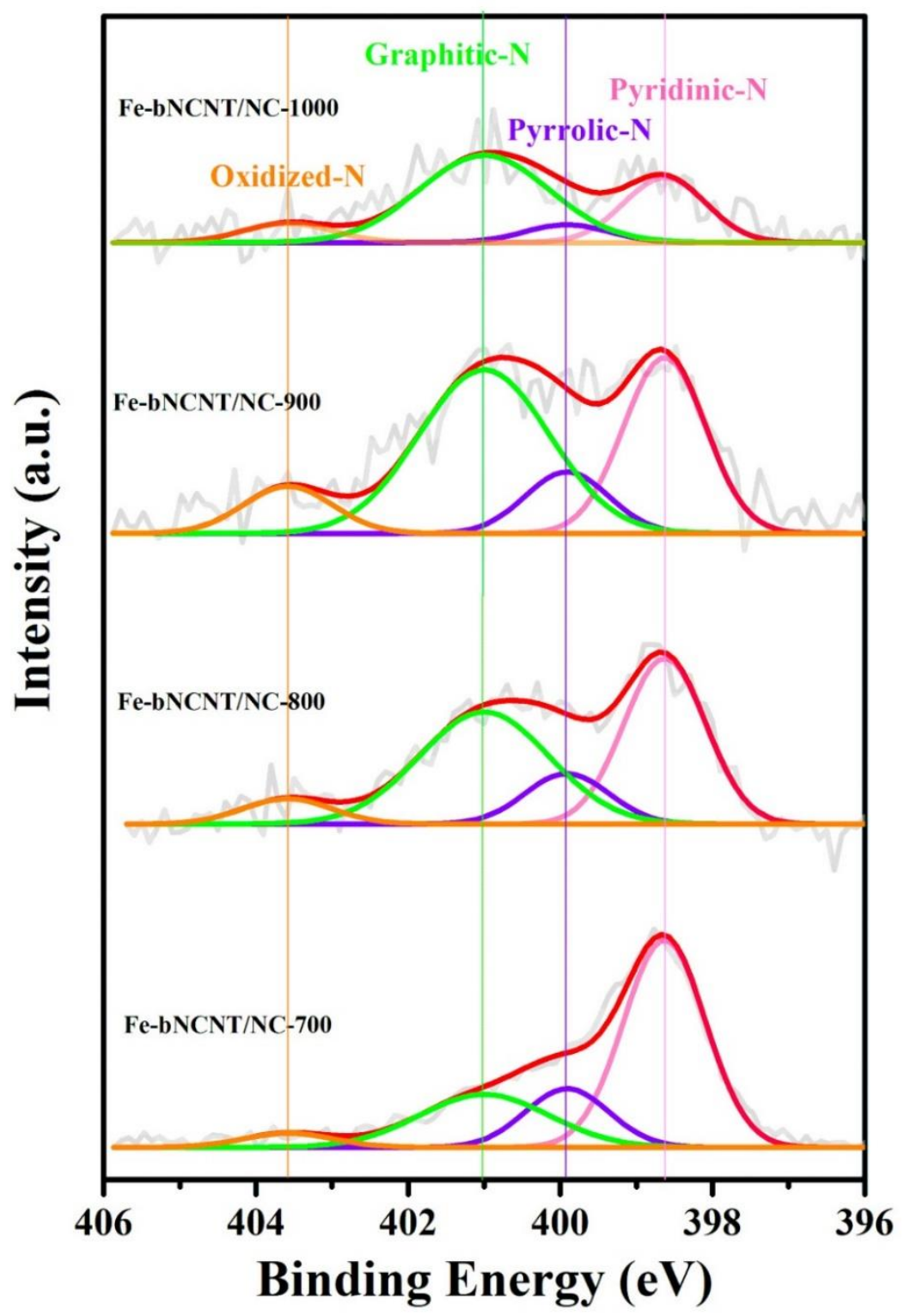

Figure S13. XPS spectra high-resolution of N1s spectra of Fe-bNCNT/NC- $T$ catalysts. 


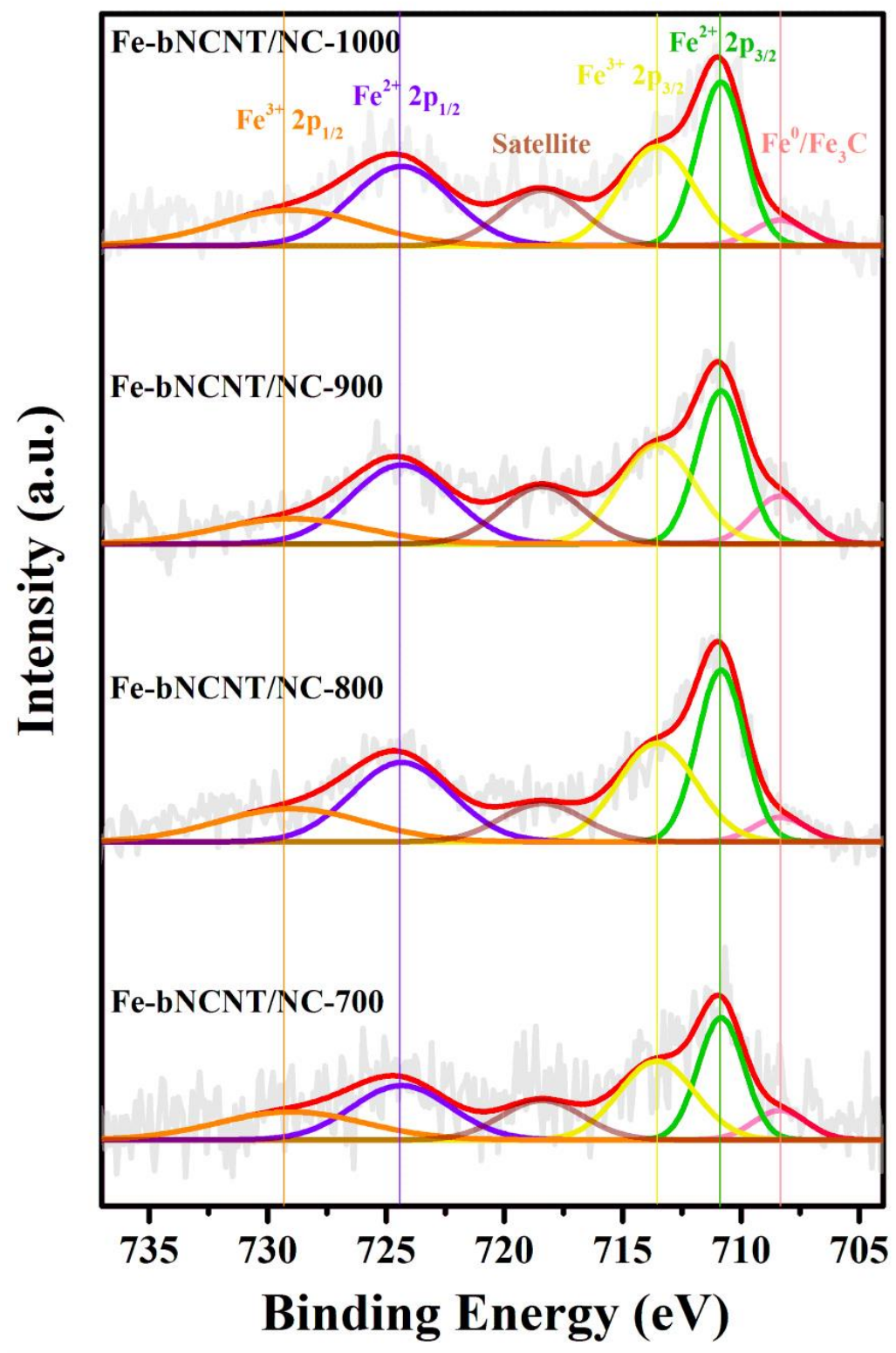

Figure S14. XPS spectra high-resolution of Fe2p spectra of Fe-bNCNT/NC- $T$ catalysts. 


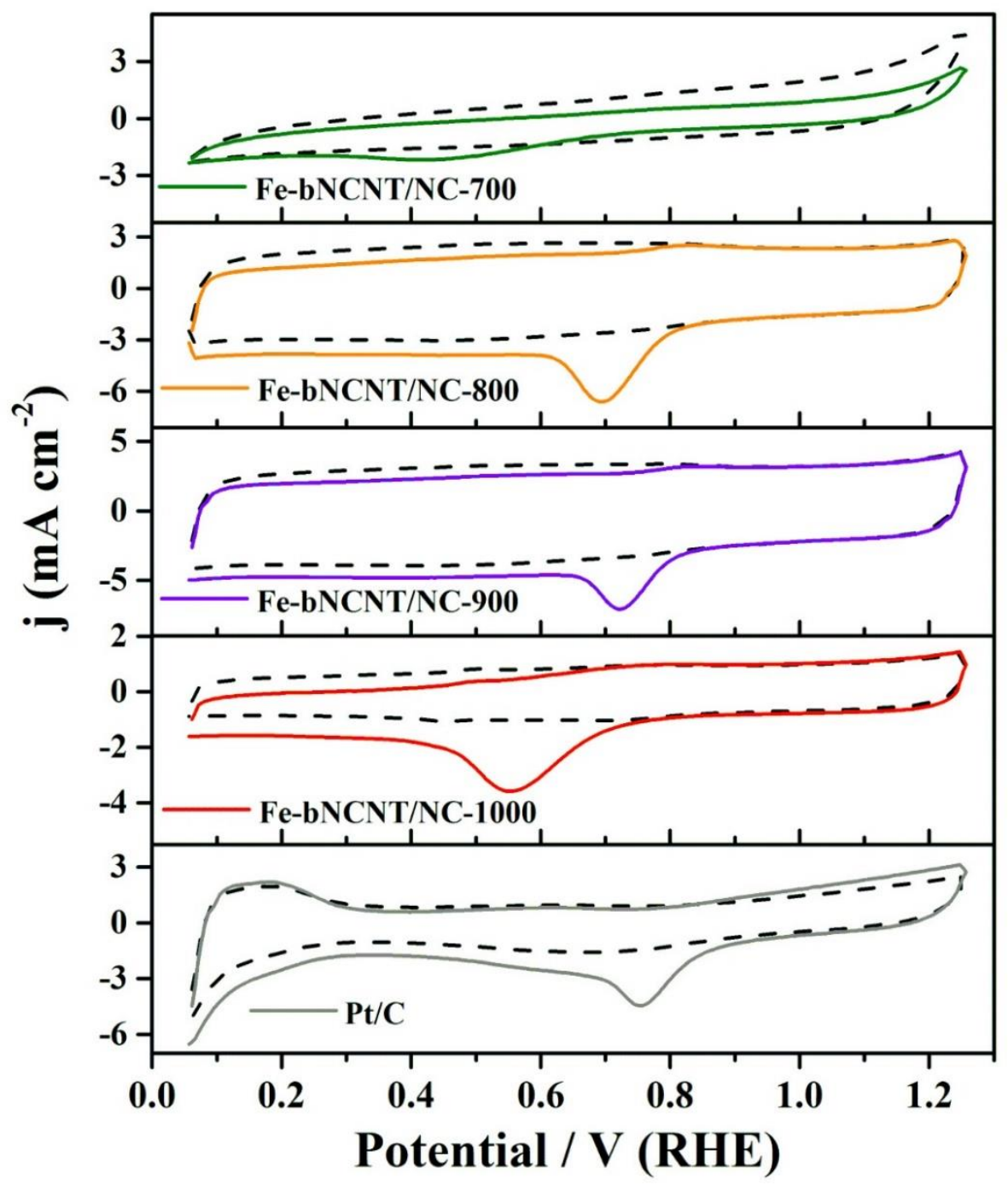

Figure S15. CV curves of Fe-bNCNT/NC-T catalysts, and $\mathrm{Pt} / \mathrm{C}$ in $\mathrm{Ar}$ (dash line)/ $\mathrm{O}_{2}$ (solid line) saturated $0.1 \mathrm{M} \mathrm{HClO}_{4}$ electrolyte. 


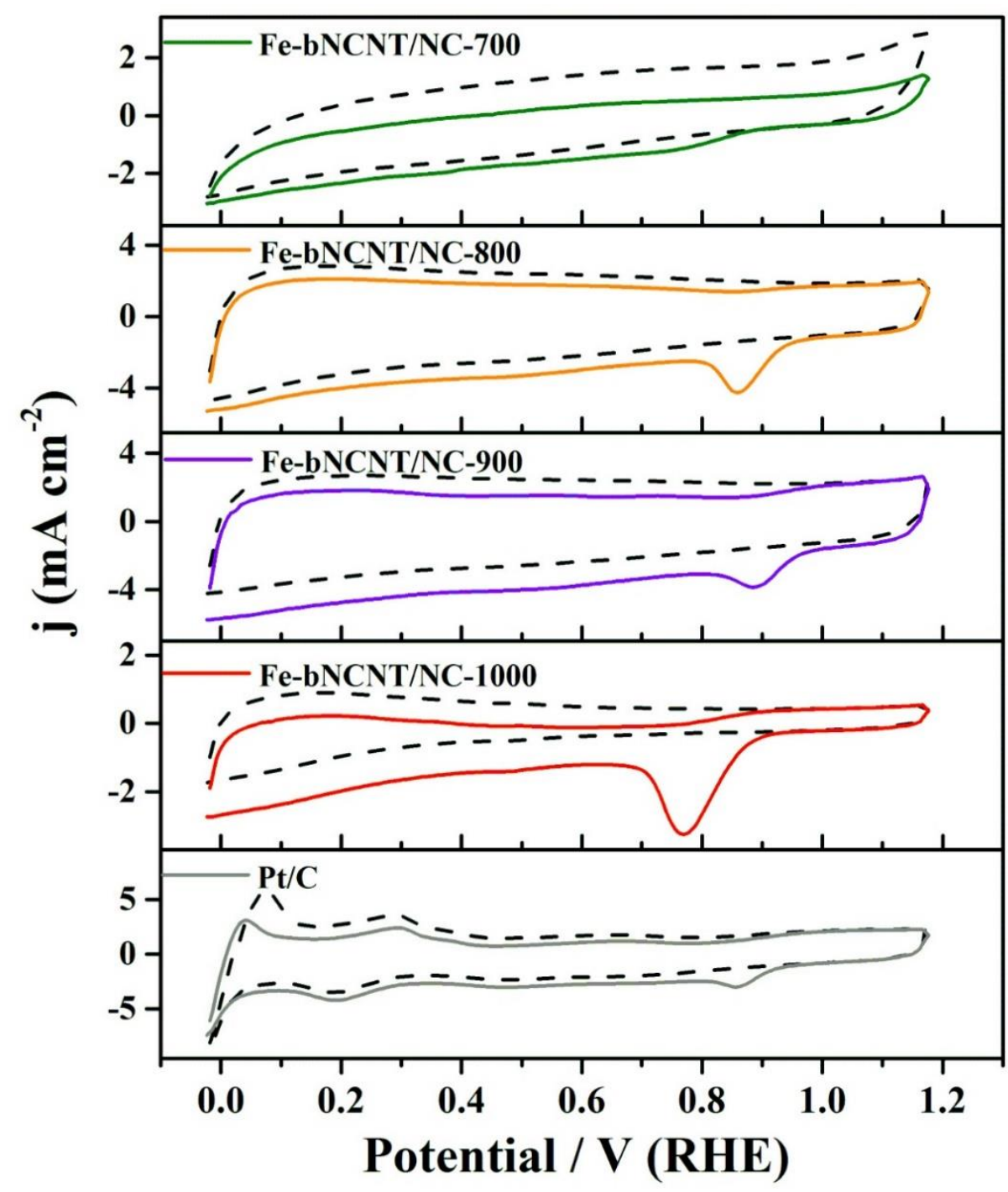

Figure S16. $\mathrm{CV}$ curves of Fe-bNCNT/NC-T, and $\mathrm{Pt} / \mathrm{C}$ in $\mathrm{Ar}$ (dash line)/ $\mathrm{O}_{2}$ (solid line) saturated $0.1 \mathrm{M} \mathrm{KOH}$ electrolyte. 

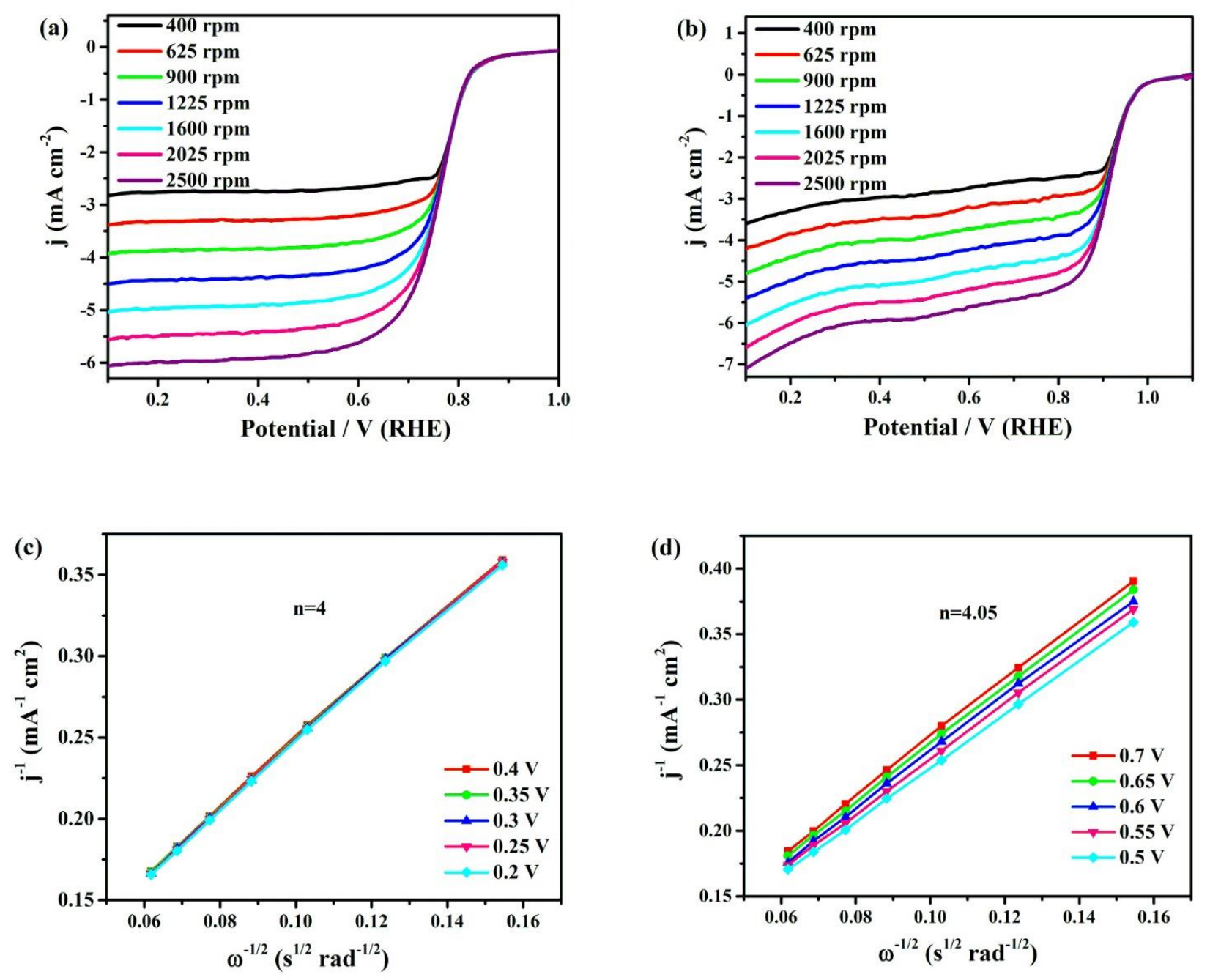

Figure S17. LSV curves of Fe-bNCNT/NC-900 at various rotating speeds in $\mathrm{O}_{2}$-saturated (a) 0.1 $\mathrm{M} \mathrm{HClO}_{4}$ electrolyte and (b) $0.1 \mathrm{M} \mathrm{KOH}$ electrolyte; corresponding K-L plots at various potentials in $\mathrm{O}_{2}$-saturated (c) $0.1 \mathrm{M} \mathrm{HClO}_{4}$ electrolyte and (d) $0.1 \mathrm{M} \mathrm{KOH}$ electrolyte. 

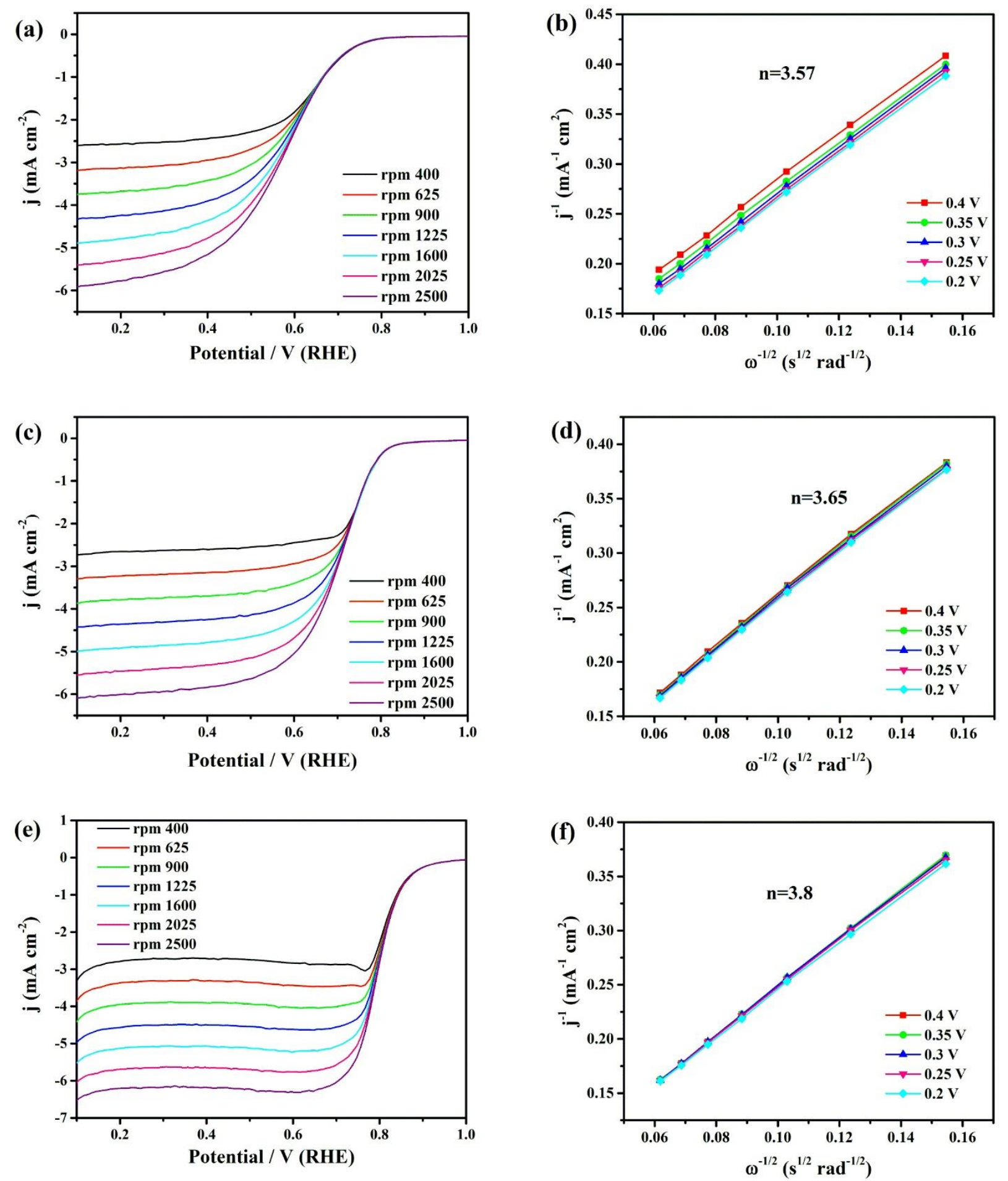

Figure S18. LSV curves at various rotating speeds and corresponding K-L plots in $\mathrm{O}_{2}$-saturated $0.1 \mathrm{M} \mathrm{HClO}_{4}$ electrolyte at various potentials of (a, b) Fe-bNCNT/NC-1000, (c, d) Fe-bNCNT/NC800 , and (e, f) $10 \mathrm{wt} \% \mathrm{Pt} / \mathrm{C}$. 

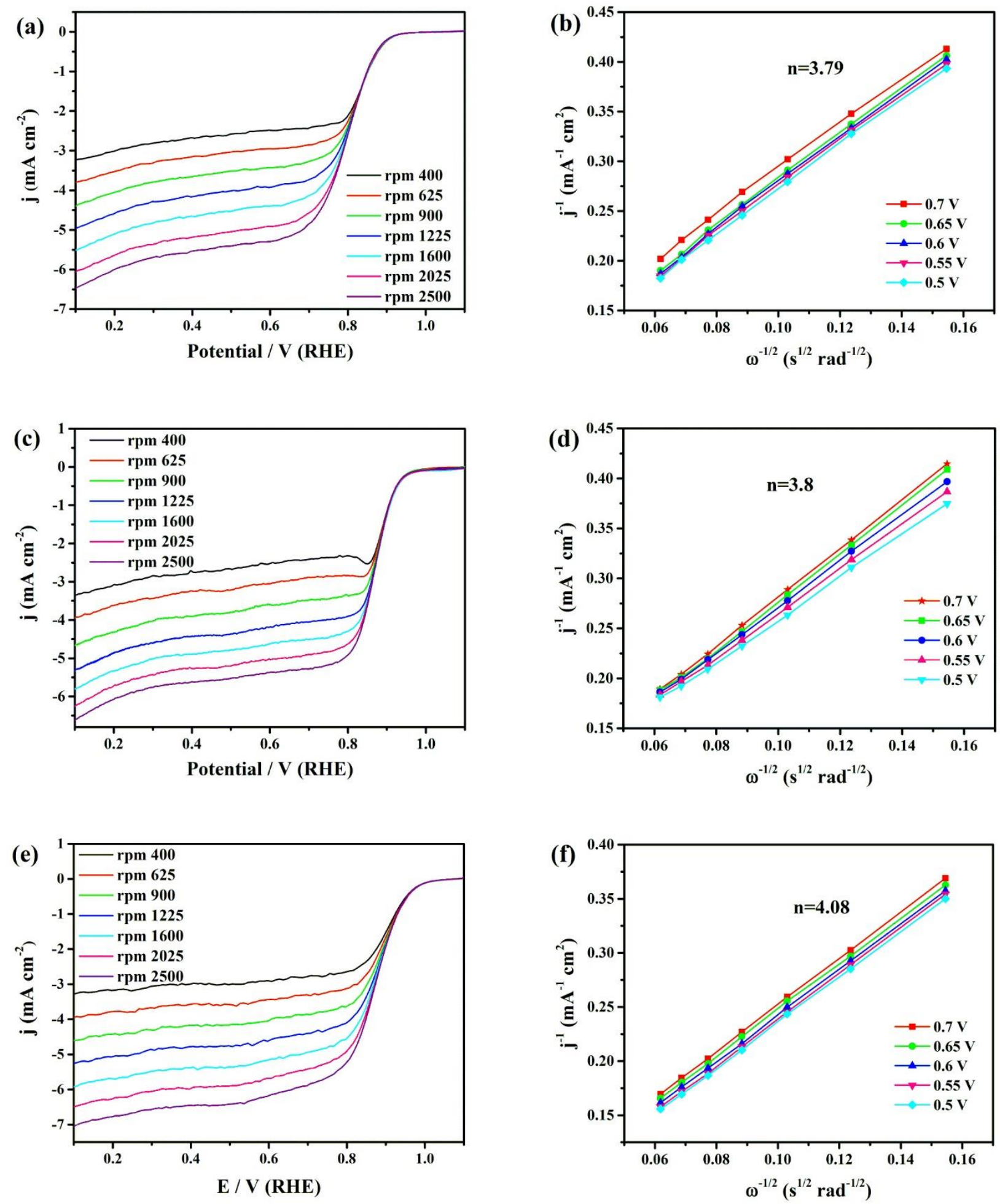

Figure S19. LSV curves at various rotating speeds and corresponding $\mathrm{K}-\mathrm{L}$ plots in $\mathrm{O}_{2}$-saturated 0.1 M KOH electrolyte at various potentials of (a, b) Fe-bNCNT/NC-1000, (c, d) Fe-bNCNT/NC800, and (e, f) 10 wt.\% Pt/C. 

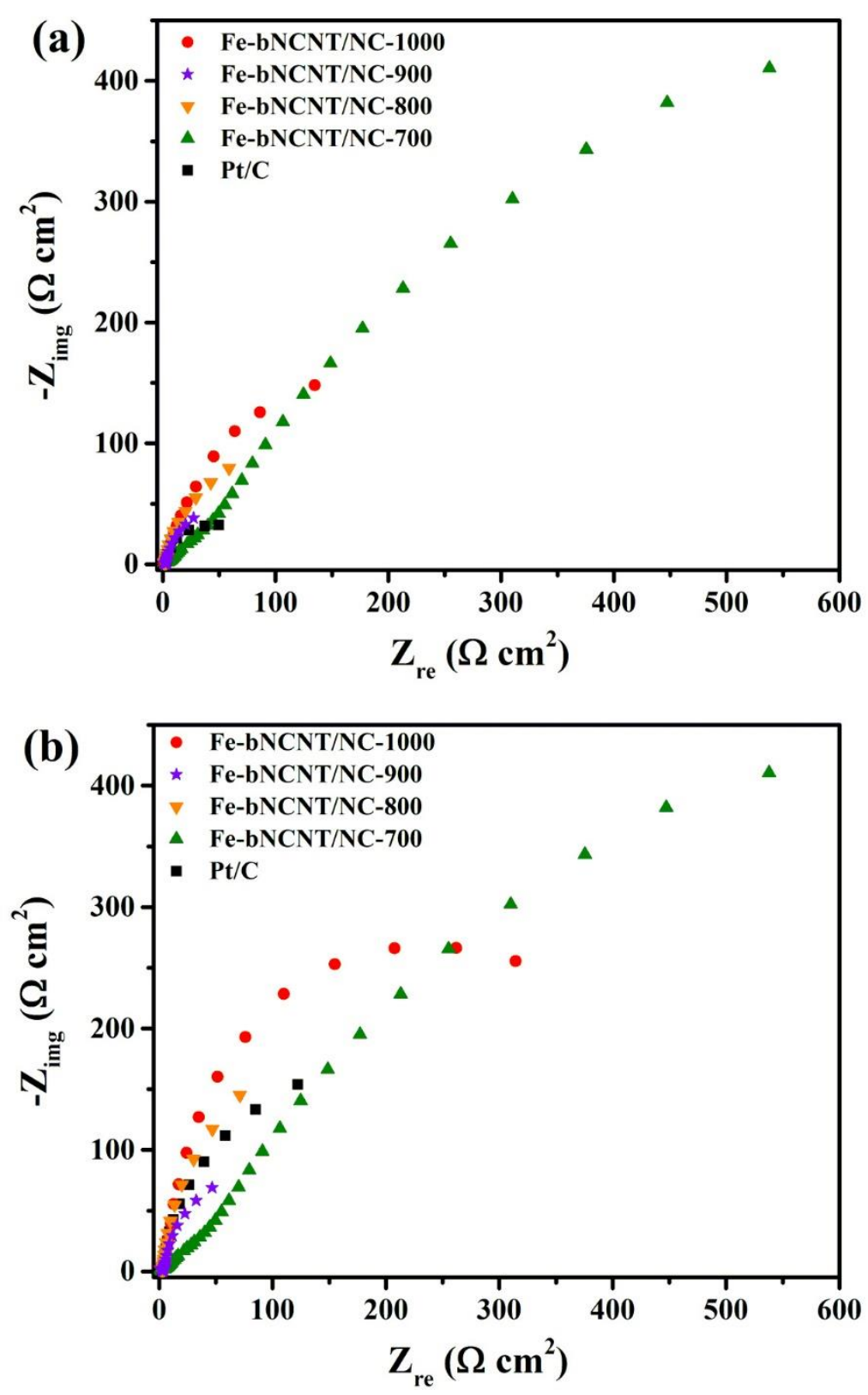

(c)

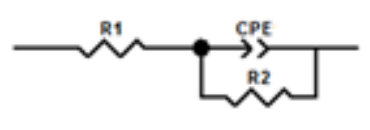

(d)

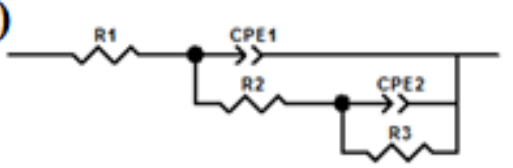

Figure S20. Electrochemical impedance spectra (EIS) of; Fe-bNCNT/NC-1000, Fe-bNCNT/NC900, Fe-bNCNT/NC-800, and Fe-bNCNT/NC-700, and $10 \mathrm{wt} \% \mathrm{Pt} / \mathrm{C}$ in (a) $0.1 \mathrm{M} \mathrm{HClO}_{4}$ and (b) $0.1 \mathrm{M} \mathrm{KOH}$. The equivalent circuit model obtained from Z-sim software for: (c) Fe-bNCNT/NC1000, Fe-bNCNT/NC-900, Fe-bNCNT/NC-800 and $10 \mathrm{wt} \%$ Pt/C, (d) Fe-bNCNT/NC-700 in both $0.1 \mathrm{M} \mathrm{HClO}_{4}$ and $0.1 \mathrm{M} \mathrm{KOH}$ electrolytes. 

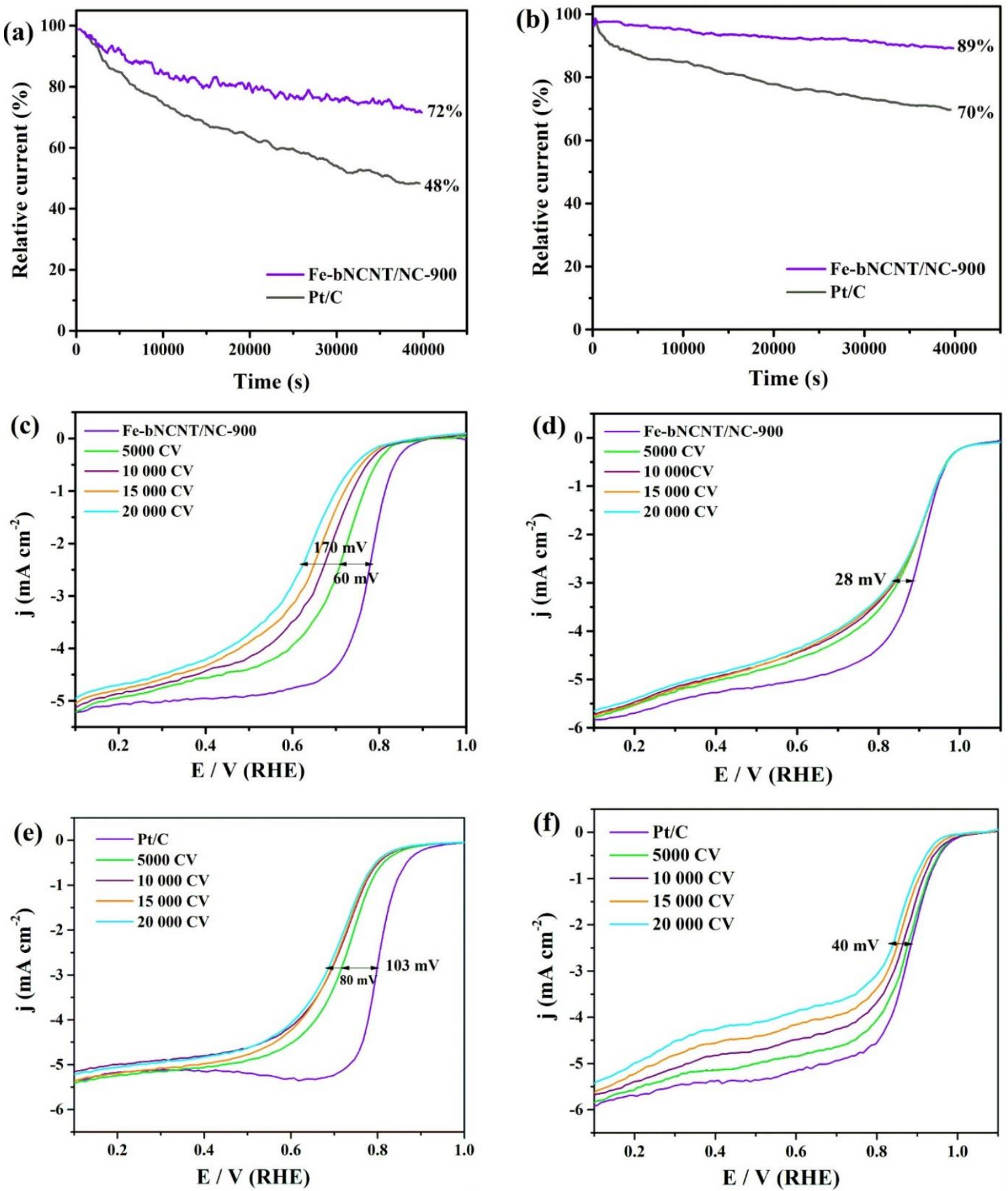

Figure S21. Durability evolution of Fe-bNCNT/NC-900 and $10 \mathrm{wt} \% \mathrm{Pt} / \mathrm{C}$ for $40,000 \mathrm{~s}$ in $\mathrm{O}_{2}-$ saturated (a) $0.1 \mathrm{M} \mathrm{HClO}_{4}$ and (b) $0.1 \mathrm{M} \mathrm{KOH}$ at $900 \mathrm{rpm}$ and $0.65 \mathrm{~V}_{\mathrm{RHE}}$. LSV curves for FebNCNT/NC-900 and 10 wt.\% Pt/C (c, e) in $0.1 \mathrm{M} \mathrm{HClO}_{4}$ electrolyte, and (d, f) in $0.1 \mathrm{M} \mathrm{KOH}$ electrolyte, respectively, before and after 5000 cycles, 10000 cycles, 15000 cycles, and 20000 cycles from $0.6 \mathrm{~V}_{\mathrm{RHE}}$ to $1.0 \mathrm{~V}_{\mathrm{RHE}}$ in $\mathrm{O}_{2}$-saturated at $1600 \mathrm{rpm}$ and $10 \mathrm{mV} \mathrm{s}^{-1}$. 


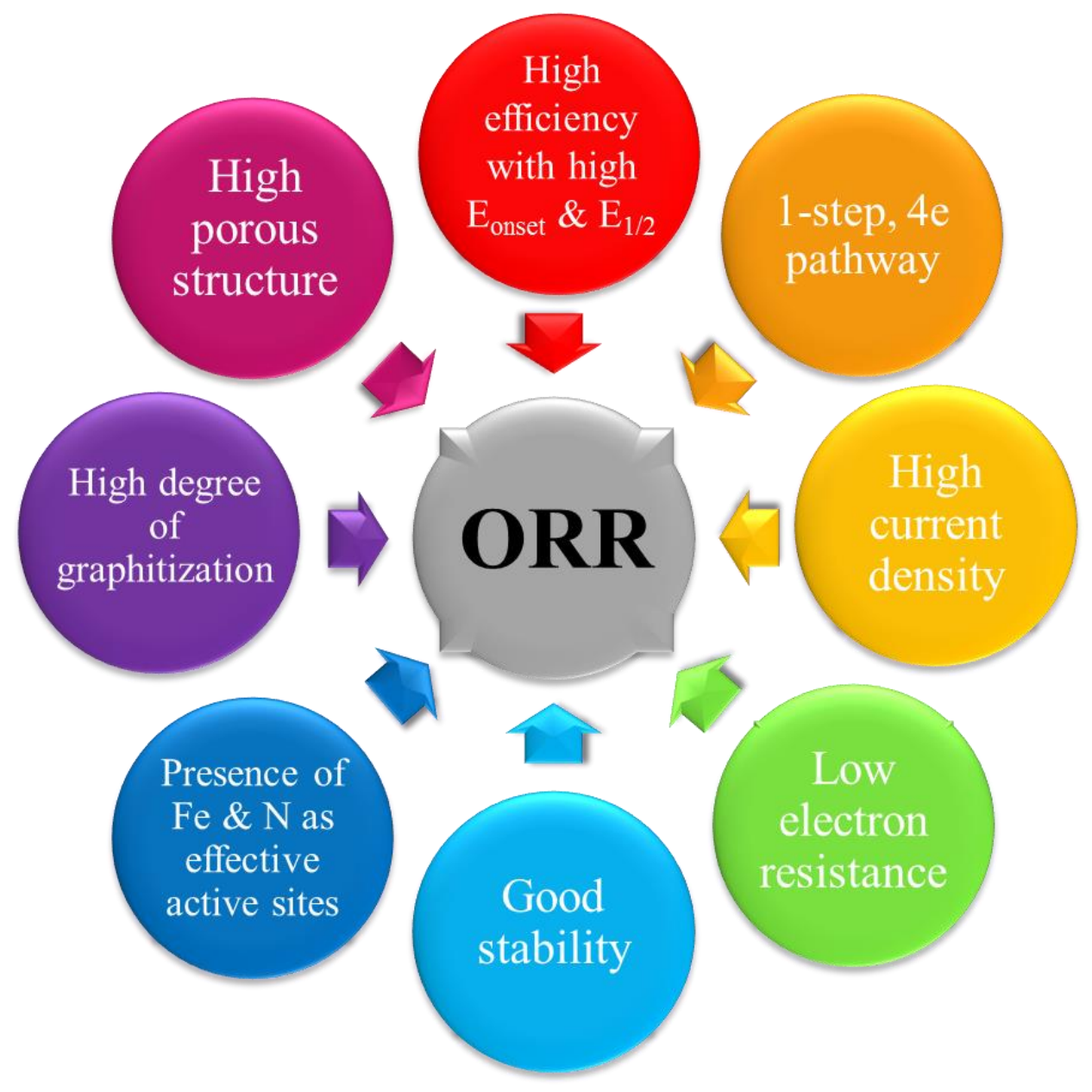

Figure S22. Critical parameters in MOF precursor that lead to an excellent NPMCs (FebNCNT/NC-900) with good ORR features.

The merit of selected $\mathrm{MOF}$ and its hybrid-composite with pyrrole and $\mathrm{FeCl}_{3}$ can be accounted to: large surface area, and high porosity as confirmed by BET analysis, high content of $\mathrm{N}$ and $\mathrm{Fe}$ (confirmed by XPS) as active sites, high thermal stability (confirmed by TGA), high crystallinity (as shown by XRD) that can stimulate the left behind organic linkers and ions to carbonaceous structure with high degree of graphitization (as Raman confirmed) during pyrolysis process. All these features led to produce catalyst with excellent ORR activity and stability like: good onset and half-wave potentials in both acidic and alkaline media, accompanied by a fast kinetic reaction 
in lower Tafel slope as well as desired one-step, 4-electron pathway in both acidic and alkaline electrolytes which are very close to Pt/C performances. Moreover, the effect of the features may be more reflected in the stability test of the optimum catalyst (Fe-bNCNT/NC-900) versus Pt/C catalyst performance. Furthermore, the comparison and some good features of this catalyst versus other as-reported catalysts were reflected in Table S7.

(a)
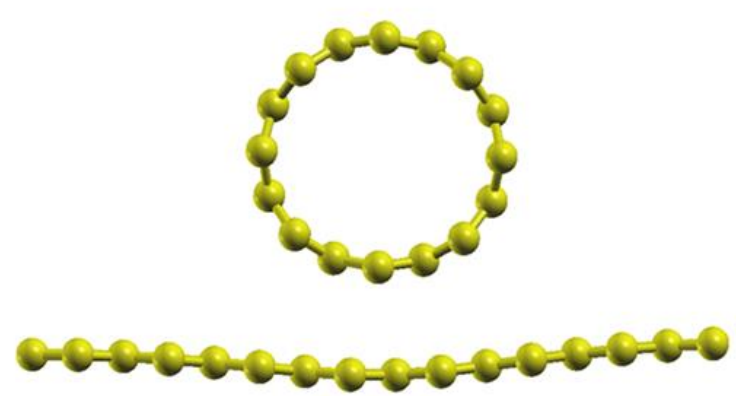

(b)

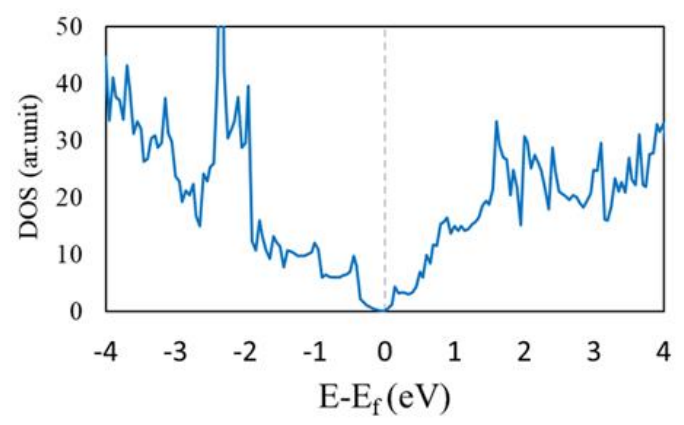

Figure S23. (a) Structure and (b) DOS plot of graphene/SWCNT. The zero-energy is set to the Fermi energy.

(a)
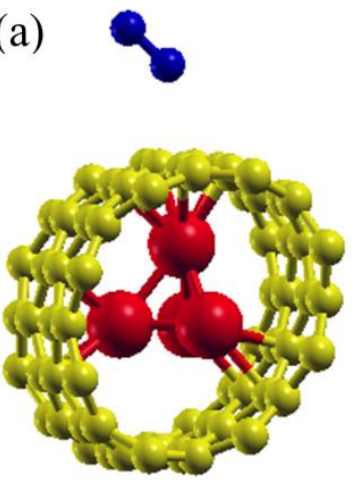

(b)
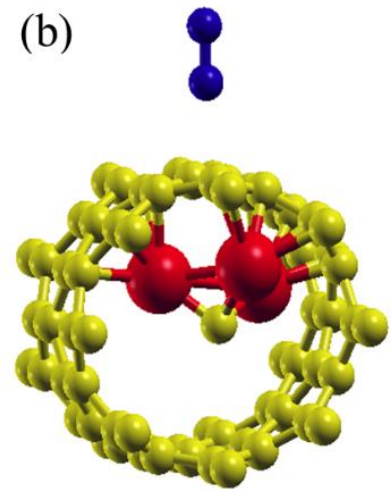

Figure S24. The adsorption of $\mathrm{O}_{2}$ on the a) $\mathrm{Fe}_{4} / \mathrm{CNT}$ and b) $\mathrm{Fe}_{3} \mathrm{C} / \mathrm{CNT}$ complexes. 
Table S1. $\mathrm{N}_{2}$ adsorption-desorption characterization of the samples before and after thermal treatment.

\begin{tabular}{lcc}
\hline Sample & $\begin{array}{c}\text { BET } \\
\left(\mathbf{m}^{\mathbf{2}} \mathbf{g}^{-\mathbf{1}}\right)\end{array}$ & $\begin{array}{c}\text { Total pore volume } \\
\left(\mathbf{c m}^{\mathbf{3}} \mathbf{g}^{-\mathbf{1}}\right)\end{array}$ \\
\hline ZIF-8 & 1901 & 0.693 \\
Fe/ZIF-8 & 1812 & 0.679 \\
$(\mathrm{Fe} / \mathrm{ZIF}-8)-\mathrm{PPy} / \mathrm{FeCl}_{3}$ & 1385 & 0.539 \\
Fe-bNCNT/NC-1000 & 344 & 0.873 \\
Fe-bNCNT/NC-900 & 554 & 0.607 \\
Fe-bNCNT/NC-800 & 543 & 0.545 \\
Fe-bNCNT/NC-700 & 143 & 0.199 \\
\hline
\end{tabular}

Table S2. Elemental composition of the precursor and the as-prepared catalysts obtained from XPS as a function of heating temperature.

\begin{tabular}{ccccccc}
\hline $\begin{array}{c}\text { Heating } \\
\text { Temperature } \\
\left({ }^{\circ} \mathbf{C}\right)\end{array}$ & \multicolumn{7}{c}{ Atomic concentration (\%) } \\
25 & $\mathbf{C}$ & $\mathbf{N}$ & $\mathbf{O}$ & $\mathbf{F e}$ & $\mathbf{Z n}$ & $\mathbf{C l}$ \\
\cline { 2 - 7 } & 55.52 & 19.07 & 16.37 & 0.59 & 6.29 & 2.07 \\
700 & 67.51 & 15.70 & 10.44 & 0.79 & 4.15 & 1.4 \\
800 & 82.98 & 7.17 & 9.52 & 0.33 & - & - \\
900 & 82.97 & 6.09 & 10.48 & 0.47 & - & - \\
1000 & 91.55 & 1.56 & 6.37 & 0.53 & - & - \\
\hline
\end{tabular}


Table S3. ORR performance of the as-synthesized catalysts collected from the LSV curves at 10 $\mathrm{mV} \mathrm{s}^{-1}$ and rotating speed of $1600 \mathrm{rpm}$ in $\mathrm{O}_{2}$-saturated $0.1 \mathrm{M} \mathrm{HClO}_{4}$ electrolyte.

\begin{tabular}{|c|c|c|c|c|}
\hline Sample & $\begin{array}{l}\text { Onset potential } \\
\text { (V vs. RHE) }\end{array}$ & $\begin{array}{l}\text { Half wave } \\
\text { potential } \\
\text { (V vs. RHE) }\end{array}$ & 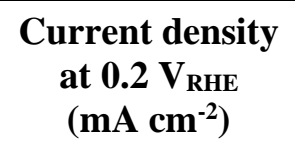 & $\begin{array}{l}\text { Tafel slope } \\
\left(\mathrm{mV} \mathrm{dec}^{-1}\right)\end{array}$ \\
\hline Fe-bNCNT/NC-1000 & $0.811 \pm 0.013$ & $0.582 \pm 0.007$ & -4.78 & 104 \\
\hline Fe-bNCNT/NC-900 & $0.900 \pm 0.010$ & $0.770 \pm 0.006$ & -5.02 & 75 \\
\hline Fe-bNCNT/NC-800 & $0.842 \pm 0.014$ & $0.711 \pm 0.007$ & -4.91 & 77 \\
\hline Fe-bNCNT/NC-700 & $0.801 \pm 0.017$ & $0.452 \pm 0.008$ & -2.86 & 175 \\
\hline $\mathrm{Pt} / \mathrm{C}(10 \%)$ & $0.950 \pm 0.013$ & $0.800 \pm 0.009$ & -5.12 & 79 \\
\hline
\end{tabular}

Table S4. ORR performance of the as-synthesized catalysts collected from the LSV curves at 10 $\mathrm{mV} \mathrm{s}^{-1}$ and rotating speed of $1600 \mathrm{rpm}$ in $\mathrm{O}_{2}$-saturated $0.1 \mathrm{M} \mathrm{KOH}$ electrolyte.

\begin{tabular}{|c|c|c|c|c|}
\hline Sample & $\begin{array}{l}\text { Onset potential } \\
\text { (V vs. RHE) }\end{array}$ & $\begin{array}{c}\text { Half wave } \\
\text { potential } \\
\text { (V vs. RHE) }\end{array}$ & 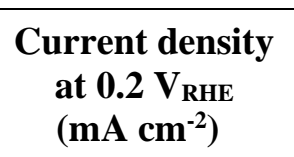 & $\begin{array}{l}\text { Tafel slope } \\
\left(\mathrm{mV} \operatorname{dec}^{-1}\right)\end{array}$ \\
\hline Fe-bNCNT/NC-1000 & $0.923 \pm 0.008$ & $0.814 \pm 0.005$ & -5.07 & 74 \\
\hline Fe-bNCNT/NC-900 & $1.020 \pm 0.007$ & $0.920 \pm 0.004$ & -5.52 & 57 \\
\hline Fe-bNCNT/NC-800 & $0.961 \pm 0.008$ & $0.882 \pm 0.006$ & -5.33 & 59 \\
\hline Fe-bNCNT/NC-700 & $0.902 \pm 0.011$ & $0.701 \pm 0.006$ & -3.75 & 135 \\
\hline $\mathrm{Pt} / \mathrm{C}(10 \%)$ & $1.000 \pm 0.009$ & $0.880 \pm 0.007$ & -5.70 & 72 \\
\hline
\end{tabular}


Table S5. The obtained values after fitting electrochemical impedance spectra (EIS) of; FebNCNT/NC-1000, Fe-bNCNT/NC-900, Fe-bNCNT/NC-800, and Fe-bNCNT/NC-700, and 10 wt $\% \mathrm{Pt} / \mathrm{C}$ in $0.1 \mathrm{M} \mathrm{HClO}_{4}$ and $0.1 \mathrm{M} \mathrm{KOH}$ with the equivalent circuit model.

\begin{tabular}{|c|c|c|c|c|c|c|}
\hline \multirow[b]{2}{*}{ Sample } & \multicolumn{3}{|c|}{$\mathrm{O.1}_{\mathrm{M} \mathrm{HClO}}$} & \multicolumn{3}{|c|}{$0.1 \mathrm{M} \mathrm{KOH}$} \\
\hline & $\begin{array}{c}R_{\mathrm{s}} \\
\left(\Omega \mathrm{cm}^{2}\right)\end{array}$ & $\begin{array}{c}R_{\mathrm{ct}} \\
\left(\mathbf{\Omega ~ c m}^{2}\right)\end{array}$ & $\begin{array}{c}\mathrm{C}_{\mathrm{dI}} \\
\left(\mathrm{mF} \mathrm{cm}^{-2}\right)\end{array}$ & $\begin{array}{c}\mathbf{R}_{\mathrm{s}} \\
\left(\mathbf{\Omega} \mathbf{c m}^{2}\right)\end{array}$ & $\begin{array}{c}R_{\mathrm{ct}} \\
\left(\mathbf{\Omega ~ c m}^{2}\right)\end{array}$ & $\begin{array}{c}\mathbf{C}_{\mathrm{dI}} \\
\left(\mathrm{mF} \mathrm{cm}^{-2}\right)\end{array}$ \\
\hline $\mathrm{Pt} / \mathrm{C}(10 \%)$ & 2.21 & 66.65 & 11 & 2.39 & 279.8 & 12 \\
\hline $\begin{array}{c}\text { Fe-bNCNT/NC- } \\
1000\end{array}$ & 2.23 & 237.4 & 7.1 & 3.59 & 536.7 & 3.1 \\
\hline $\begin{array}{c}\text { Fe-bNCNT/NC- } \\
900\end{array}$ & 2.36 & 70.05 & 47 & 3.25 & 128.8 & 27.7 \\
\hline $\begin{array}{c}\text { Fe-bNCNT/NC- } \\
800\end{array}$ & 2.24 & 127.16 & 22 & 3.08 & 337.1 & 15 \\
\hline $\begin{array}{c}\text { Fe-bNCNT/NC- } \\
700\end{array}$ & 1.87 & 1567 & 1.8 & 1.98 & 2981 & 0.8 \\
\hline
\end{tabular}

Table S6. HOMO, LOMO, energy gap, adsorption energy, and O-O bond length after $\mathrm{O}_{2}$ adsorption.

\begin{tabular}{cccccc}
\hline Complex & $\begin{array}{c}\text { HOMO } \\
(\mathbf{e V})\end{array}$ & $\begin{array}{c}\text { LUMO } \\
(\mathbf{e V})\end{array}$ & $\begin{array}{c}\text { Energy gap } \\
(\mathbf{e V})\end{array}$ & $\begin{array}{c}\text { Adsorption energy } \\
(\mathbf{e V})\end{array}$ & $\begin{array}{c}\text { O-O bond length } \\
(\AA)\end{array}$ \\
\hline $\mathrm{Fe}_{3} \mathrm{C} / \mathrm{CNT}$ & -2.185 & -1.982 & 0.203 & -0.45 & 1.25 \\
$\mathrm{Fe}_{4} / \mathrm{CNT}$ & -2.187 & -2.177 & 0.010 & -0.33 & 1.24 \\
\hline
\end{tabular}


Table S7. Comparison of the ORR performance of the recently reported MOF-derived carbon-based catalysts

\begin{tabular}{|c|c|c|c|c|c|c|c|c|c|c|}
\hline Catalyst & Precursors & $\begin{array}{l}\text { Surface } \\
\text { area } \\
\left(\mathbf{m}^{2} \mathbf{g}^{-1}\right)\end{array}$ & Morphology & Electrolyte & $\begin{array}{c}\text { Eonset } \\
\left(\mathbf{V}_{\text {RHE }}\right)\end{array}$ & $\begin{array}{c}\mathbf{E}_{1 / 2} \\
\left(\mathbf{V}_{\text {RHE }}\right)\end{array}$ & $\begin{array}{l}\text { Tafel slope } \\
\left(\mathrm{mV} \operatorname{dec}^{-1}\right)\end{array}$ & $\begin{array}{l}\text { Number } \\
\text { of } \\
\text { electron }\end{array}$ & Stability test & Ref. \\
\hline $\begin{array}{c}\text { Fe-bNCNT/NC- } \\
900\end{array}$ & $\begin{array}{c}\text { ZIF-8, Fe(acac })_{3}, \\
\text { Pyrrole }\end{array}$ & 554 & $\begin{array}{l}\text { Iron based nanoparticles } \\
\text { enwrapped in porous } \\
\text { bamboo-like N-doped } \\
\text { carbon nanotubes grown } \\
\text { on an N-doped sheet-like } \\
\text { carbon. }\end{array}$ & $\begin{array}{l}0.1 \mathrm{M} \mathrm{KOH} \\
0.1 \mathrm{M} \mathrm{HClO}_{4}\end{array}$ & 0.900 & 0.920 & 57 & $\sim 4.0$ & $\begin{array}{l}89 \% \text { and } 72 \% \text { retention of } \mathrm{j} \text { in } \\
\text { current-time chronoamperometry } \\
\text { through } 40000 \mathrm{~s} \text { in alkaline and } \\
\text { acidic solution, respectively. } \\
28 \mathrm{mV} \text { and } 170 \mathrm{mV} \text { negative shift } \\
\text { (half wave) in ADT through } 20000 \\
\text { cycles in alkaline and acidic } \\
\text { solution, respectively. }\end{array}$ & $\begin{array}{l}\text { This } \\
\text { work }\end{array}$ \\
\hline $\begin{array}{c}\text { Fe,Co,N } \\
\text { CNP(0.3) }\end{array}$ & $\begin{array}{c}\text { ZIF-67 } \\
\text { Bimetallic }(\mathrm{Zn} / \mathrm{Fe}) \\
\mathrm{FeSO}_{4} \cdot 7 \mathrm{H}_{2} \mathrm{O}\end{array}$ & 422 & $\begin{array}{l}\text { Co and } \mathrm{Fe} \text { nanoparticles } \\
\text { dispersed on porous N- } \\
\text { doped carbon framework. }\end{array}$ & $0.1 \mathrm{M} \mathrm{KOH}$ & 0.979 & 0.875 & 75 & 3.96 & $\begin{array}{l}90.2 \% \text { and } 72.1 \% \text { retention of } j \text { in } \\
\text { current-time chronoamperometry } \\
\text { through } 10000 \text { s in alkaline and } \\
\text { acidic solution, respectively. }\end{array}$ & 1 \\
\hline Fe,N-HPCC & $\begin{array}{c}\text { ZIF-7 } \\
\text { Bimetallic }(\mathrm{Zn} / \mathrm{Fe}) \\
\mathrm{FeSO}_{4} .7 \mathrm{H}_{2} \mathrm{O}\end{array}$ & 817 & $\begin{array}{l}\text { Iron- and nitrogen co- } \\
\text { doped hierarchically } \\
\text { porous carbon cylinders. }\end{array}$ & $0.1 \mathrm{M} \mathrm{KOH}$ & 0.972 & 0.898 & 61 & 3.99 & $\begin{array}{l}94.9 \% \text { and } 81.1 \% \text { retention of } j \text { in } \\
\text { current-time chronoamperometry } \\
\text { through } 10000 \mathrm{~s} \text { in alkaline and } \\
\text { acidic solution, respectively. }\end{array}$ & 2 \\
\hline FeNC-20-1000 & $\begin{array}{c}\text { ZIF-7 } \\
\text { Bimetallic }(\mathrm{Zn} / \mathrm{Fe}) \\
\mathrm{FeSO}_{4} .7 \mathrm{H}_{2} \mathrm{O}\end{array}$ & N/A & $\begin{array}{l}\text { A hybrid of porous carbon } \\
\text { nanosheets and nanotubes } \\
\text { with no clear metallic } \\
\text { nanoparticles. }\end{array}$ & $0.1 \mathrm{M} \mathrm{KOH}$ & 1.04 & 0.88 & 49 & 3.99 & $\begin{array}{l}89 \% \text { and } 75 \% \text { retention of } j \text { in } \\
\text { current-time chronoamperometry } \\
\text { through } 20000 \mathrm{~s} \text { in alkaline and } \\
\text { acidic solution, respectively. }\end{array}$ & 3 \\
\hline C-Fe-Z8-Ar & $\begin{array}{c}\text { ZIF-8 } \\
\text { Bimetallic }(\mathrm{Zn} / \mathrm{Fe}) \\
\text { Span } 80 \\
\text { Tween } 80 \\
\mathrm{FeSO}_{4}\end{array}$ & 969 & $\begin{array}{l}\text { Well-dispersed atomic } \mathrm{Fe} \\
\text { in porous } \mathrm{N} \text {-doped carbon } \\
\text { matrix. }\end{array}$ & $0.1 \mathrm{M} \mathrm{HClO}_{4}$ & 0.95 & 0.82 & 70 & 3.98 & $\begin{array}{l}40 \mathrm{mV} \text { negative shift (half wave) in } \\
\text { ADT through } 10000 \text { cycles in acidic } \\
\text { solution. }\end{array}$ & 4 \\
\hline
\end{tabular}


$\operatorname{Bimetallic}(\mathrm{Zn} / \mathrm{Fe})$ matrix.

Iron salt

\begin{tabular}{|c|c|c|c|c|c|c|c|c|c|c|}
\hline $\begin{array}{c}\text { C-FeZIF-900- } \\
0.84\end{array}$ & $\begin{array}{c}\text { ZIF-8 } \\
\text { Bimetallic( } \mathrm{Zn} / \mathrm{Fe}) \\
\text { Iron (III) } \\
\text { acetylacetonate }\end{array}$ & 926 & $\begin{array}{l}\text { Well-dispersed atomic } \mathrm{Fe} \\
\text { in porous } \mathrm{N} \text {-doped carbon } \\
\text { matrix. }\end{array}$ & $0.1 \mathrm{M} \mathrm{HClO}_{4}$ & 0.90 & 0.77 & N/A & 3.4 & $\begin{array}{l}95 \% \text { and } 93 \% \text { retention of } j \text { in } \\
\text { current-time chronoamperometry } \\
\text { through } 20000 \mathrm{~s} \text { in alkaline and } \\
\text { acidic solution, respectively. }\end{array}$ & 6 \\
\hline $\begin{array}{c}\text { FePhenMOF- } \\
\text { ArNH }_{3}\end{array}$ & $\begin{array}{c}\text { ZIF-8 } \\
\text { 1,10- } \\
\text { phenanthroline } \\
\text { monohydrate } \\
\text { Iron(II) acetate }\end{array}$ & $\sim 1360$ & $\begin{array}{l}\text { Interconnected hollow } \\
\text { structures with no } \\
\text { metallic aggregates. }\end{array}$ & $0.1 \mathrm{M} \mathrm{HClO}_{4}$ & 0.98 & 0.78 & N/A & N/A & N/A & 7 \\
\hline $\begin{array}{c}\text { FePhen@MOF- } \\
\qquad \mathrm{ArNH}\end{array}$ & $\begin{array}{c}\text { ZIF-8 } \\
\text { 1,10- } \\
\text { phenanthroline } \\
\text { monohydrate } \\
\text { Iron(II) acetate }\end{array}$ & 1200 & $\begin{array}{l}\mathrm{Fe} / \mathrm{Fe}_{\mathrm{x}} \mathrm{C} \text { nanoparticles } \\
\text { encapsulated into distinct } \\
\text { bamboo-like joints. }\end{array}$ & $\begin{array}{c}0.1 \mathrm{M} \mathrm{KOH} \\
0.1 \mathrm{M} \mathrm{HClO}_{4}\end{array}$ & 1.03 & 0.86 & N/A & N/A & $\begin{array}{l}\text { Stability test was performed in fuel } \\
\text { cell test station through } 10000 \\
\text { potential cycles (using } 3 \mathrm{~s} \text { hold and } \\
\text { pulses between } 0.6 \text { and } 1.0 \mathrm{~V} \text { under } \\
\text { nitrogen flow). }\end{array}$ & 8 \\
\hline Fe/N-GPC & $\begin{array}{c}\text { MIL-101- } \mathrm{NH}_{2} \\
\text { DCD } \\
\mathrm{FeCl}_{3}\end{array}$ & 1207 & $\begin{array}{l}\mathrm{Fe}_{3} \mathrm{C} \text { nanoparticles } \\
\text { accompanied with well- } \\
\text { dispersed atomic Fe in } \\
\text { macro/meso/micro porous } \\
\text { N-doped carbon matrix. }\end{array}$ & $0.5 \mathrm{M} \mathrm{H}_{2} \mathrm{SO}_{4}$ & 0.967 & 0.847 & N/A & $\sim 4.0$ & $\begin{array}{l}95 \% \text { retention of } j \text { in current-time } \\
\text { chronoamperometry through } 20000 \\
\mathrm{~s} \text { in alkaline solution. }\end{array}$ & 9 \\
\hline $\mathrm{FeCo14Zn23/N/C}$ & $\begin{array}{c}\text { ZIF-67 } \\
\text { ZIF-8 } \\
\text { 1,10- } \\
\text { phenanthroline } \\
\text { monohydrate } \\
\text { Iron(II) acetate }\end{array}$ & 19 & $\begin{array}{l}\text { A hybrid of amorphous } \\
\text { carbon matrix, cobalt } \\
\text { nanoparticles and } \\
\text { bamboo-like nanotubes. }\end{array}$ & $0.1 \mathrm{M} \mathrm{HClO}_{4}$ & 0.762 & 0.605 & N/A & $\sim 3.9$ & $\begin{array}{l}12 \mathrm{mV} \text { positive shift (half wave) in } \\
\text { ADT through } 1000 \text { cycles in acidic } \\
\text { solution. } \\
\text { Improvement of } j \text { in current-time } \\
\text { chronoamperometry from } 6000 \mathrm{~s} \text { to } \\
30000 \mathrm{~s} \text { in acidic solution. }\end{array}$ & 10 \\
\hline
\end{tabular}




\begin{tabular}{|c|c|c|c|c|c|c|c|c|c|c|}
\hline $\mathrm{Fe}_{3} \mathrm{C} / \mathrm{bNCNT}$ & $\begin{array}{l}\mathrm{ZIF}-8 \\
\mathrm{FeCl}_{3} .6 \mathrm{H}_{2} \mathrm{O} \\
\text { Pyrrole }\end{array}$ & 185 & $\begin{array}{l}\mathrm{Fe}_{3} \mathrm{C} \text { nanoparticles } \\
\text { encapsulated in N-doped } \\
\text { bamboo-like carbon } \\
\text { nanotubes (b-NCNTs). }\end{array}$ & $0.5 \mathrm{M} \mathrm{H}_{2} \mathrm{SO}_{4}$ & N/A & N/A & 71.2 & $\sim 4.0$ & $\begin{array}{l}\text { High retention of } j \text { in current-time } \\
\text { chronoamperometry through } 252000 \\
\mathrm{~s} \text { in alkaline and acidic solution. }\end{array}$ & 11 \\
\hline $\mathrm{Fe}_{3} \mathrm{C} / \mathrm{NCNF}$ & $\begin{array}{l}\text { MIL-88B-NH} 2 \\
\text { PAN }\end{array}$ & 557 & $\begin{array}{l}\text { Ultrafine } \mathrm{Fe}_{3} \mathrm{C} \\
\text { nanoparticles } \\
\text { encapsulated into carbon } \\
\text { nanofibers with } \\
\text { meso/microporous } \\
\text { architecture. }\end{array}$ & $\begin{array}{l}0.1 \mathrm{M} \mathrm{KOH} \\
0.5 \mathrm{M} \mathrm{H}_{2} \mathrm{SO}_{4}\end{array}$ & 1.012 & 0.873 & N/A & $\begin{array}{l}\sim 3.8 \\
\text { N/A }\end{array}$ & $\begin{array}{l}73 \% \text { retention of } j \text { in current-time } \\
\text { chronoamperometry through } 30000 \\
\text { s in alkaline solution. }\end{array}$ & 12 \\
\hline $\mathrm{Fe}_{3} \mathrm{C} @ \mathrm{~N}-\mathrm{CNT}$ & $\begin{array}{l}\text { MIL-88B } \\
\text { ZIF-8 } \\
\text { PVP }\end{array}$ & 377 & $\begin{array}{l}\mathrm{Fe}_{3} \mathrm{C} \text { nanoparticles } \\
\text { embedded into carbon } \\
\text { nanotube. }\end{array}$ & $0.1 \mathrm{M} \mathrm{KOH}$ & 0.97 & 0.85 & 78 & 3.96 & $\begin{array}{l}\sim 94 \% \text { retention of } j \text { in current-time } \\
\text { chronoamperometry through } 15000 \\
\text { s in alkaline solution. }\end{array}$ & 13 \\
\hline $\mathrm{Fe} / \mathrm{NC800}$ & $\begin{array}{l}\text { MIL-88c-Fe } \\
\text { DCDA }\end{array}$ & $237-289$ & $\begin{array}{l}\mathrm{Fe} / \mathrm{Fe}_{3} \mathrm{C} \text { nanoparticles } \\
\text { embedded into } \mathrm{N} \text {-doped } \\
\text { porous carbon. }\end{array}$ & $0.1 \mathrm{M} \mathrm{KOH}$ & 0.99 & 0.86 & 61 & 3.67 & $\begin{array}{l}77 \mathrm{mV} \text { negative shift (half wave) in } \\
\text { ADT through } 10000 \text { cycles in } \\
\text { alkaline solution. }\end{array}$ & 14 \\
\hline $\begin{array}{c}\text { MIL-5 DCD- } \\
800\end{array}$ & $\begin{array}{l}\text { MIL-101(Fe) } \\
\text { DCDA }\end{array}$ & 452.33 & $\begin{array}{l}\text { Fe-based nanoparticles } \\
\text { wrapped within graphene } \\
\text { structure. }\end{array}$ & $0.1 \mathrm{M} \mathrm{KOH}$ & 1.08 & 0.86 & N/A & 3.93 & $\begin{array}{l}\text { Retention } 98 \% \text { of the initial current } \\
\text { density in ADT through } 2000 \text { cycles } \\
\text { in alkaline solution. }\end{array}$ & 15 \\
\hline $\begin{array}{l}\text { MOF-253-Fe- } \\
\text { phen-C }\end{array}$ & $\begin{array}{c}\mathrm{MOF}-253 \\
\mathrm{FeCl}_{2} \\
1,10- \\
\text { phenanthroline }\end{array}$ & 538 & $\begin{array}{l}\mathrm{Fe}_{3} \mathrm{C} \text { nanoparticles } \\
\text { wrapped by hollow } \\
\text { carbon spheres. }\end{array}$ & $0.1 \mathrm{M} \mathrm{HClO}_{4}$ & $\begin{array}{l}20 \mathrm{mV} \\
<\mathrm{Pt} / \mathrm{C}\end{array}$ & $\begin{array}{l}30 \mathrm{mV} \\
<\mathrm{Pt} / \mathrm{C}\end{array}$ & N/A & 4 & $\begin{array}{l}93 \% \text { and } 88 \% \text { retention of } j \text { in } \\
\text { current-time chronoamperometry } \\
\text { through } 50000 \mathrm{~s} \text { in alkaline and } \\
\text { acidic solution, respectively. }\end{array}$ & 16 \\
\hline $\begin{array}{c}\mathrm{Fe}_{3} \mathrm{C} @ \mathrm{NCNT} / \mathrm{N} \\
\mathrm{PC}\end{array}$ & $\begin{array}{l}\text { MIL-88B } \\
\text { Melamine }\end{array}$ & 175.1 & $\begin{array}{l}\mathrm{Fe}_{3} \mathrm{C} \text { nanorods } \\
\text { encapsulated, N-doped } \\
\text { carbon nanotubes grown } \\
\text { on N-doped porous } \\
\text { carbon sheets. }\end{array}$ & $0.5 \mathrm{M} \mathrm{H}_{2} \mathrm{SO}_{4}$ & $\sim 0.9$ & 0.71 & N/A & 3.99 & $\begin{array}{l}92 \% \text { and } 88 \% \text { retention of } \mathrm{j} \text { in } \\
\text { current-time chronoamperometry } \\
\text { through } 4000 \mathrm{~s} \text { in alkaline and acidic } \\
\text { solution, respectively. } \\
13 \mathrm{mV} \text { and } 20 \mathrm{mV} \text { positive shift } \\
\text { (half wave) in ADT through } 1000 \\
\text { cycles in alkaline and acidic } \\
\text { solution, respectively. }\end{array}$ & 17 \\
\hline
\end{tabular}




\begin{tabular}{|c|c|c|c|c|c|c|c|c|c|c|}
\hline \multirow[t]{2}{*}{ C-ZIF-8 (4/2) } & $\begin{array}{l}\text { ZIF-8 } \\
\text { AFC }\end{array}$ & 1323.7 & $\begin{array}{l}\mathrm{Fe}_{3} \mathrm{C} \text { nanoparticles within } \\
\text { mesoporous carbon. }\end{array}$ & $0.1 \mathrm{M} \mathrm{KOH}$ & N/A & N/A & N/A & 4 & \multirow[t]{2}{*}{$\begin{array}{l}12 \mathrm{mV} \text { positive shift (half wave) in } \\
\text { ADT through } 10000 \text { cycles in acidic } \\
\text { solution. }\end{array}$} & \multirow[t]{2}{*}{18} \\
\hline & & & & $0.1 \mathrm{M} \mathrm{HClO}_{4}$ & N/A & $\begin{array}{l}21 \mathrm{mV} \\
<\mathrm{Pt} / \mathrm{C}\end{array}$ & N/A & $\sim 4.0$ & & \\
\hline \multirow[t]{2}{*}{$\begin{array}{l}\text { NGPC/NCNT- } \\
900\end{array}$} & $\begin{array}{l}\text { MOF-5 } \\
\text { Nickel }\end{array}$ & 1053 & $\begin{array}{l}\text { A hybrid structure of } \\
\text { carbon particulates (CPs) } \\
\text { and nanotubes. }\end{array}$ & $0.1 \mathrm{M} \mathrm{KOH}$ & 0.926 & 0.805 & 56 & 4 & \multirow[t]{2}{*}{$\begin{array}{l}86 \% \text { retention of } j \text { in current-time } \\
\text { chronoamperometry through } 43200 \\
\text { s in alkaline solution. }\end{array}$} & \multirow[t]{2}{*}{19} \\
\hline & Urea & & & $0.5 \mathrm{M} \mathrm{H}_{2} \mathrm{SO}_{4}$ & N/A & $\begin{array}{c}100 \\
\mathrm{mV}< \\
\mathrm{Pt} / \mathrm{C}\end{array}$ & N/A & N/A & & \\
\hline $\begin{array}{l}\text { NS(3:1)-C- } \\
\text { MOF-5 }\end{array}$ & $\begin{array}{c}\text { MOF-5 } \\
\text { DMSO } \\
\text { Urea }\end{array}$ & 1091 & $\begin{array}{l}\mathrm{N} \text { and } \mathrm{S} \text { co-doped porous } \\
\text { carbon. }\end{array}$ & $0.1 \mathrm{M} \mathrm{KOH}$ & 0.972 & N/A & N/A & N/A & $\begin{array}{l}80 \% \text { retention of } j \text { in current-time } \\
\text { chronoamperometry through } 20000 \\
\mathrm{~s} \text { in alkaline solution. }\end{array}$ & 20 \\
\hline $\begin{array}{c}\text { Co-Zn-ZIF/GO- } \\
\mathbf{8 0 0}\end{array}$ & $\begin{array}{c}\text { ZIF-8 } \\
\text { ZIF-67 } \\
\text { Bimetallic(Zn/Co) } \\
\text { GO }\end{array}$ & 373 & $\begin{array}{l}\mathrm{N} \text {-doped nanoporous } \\
\text { carbon/graphene } \\
\text { sandwich-like structure } \\
\text { with distributed uniformly } \\
\text { Co nanoparticle. }\end{array}$ & $0.1 \mathrm{M} \mathrm{HClO}_{4}$ & 0.96 & N/A & N/A & 4 & $\begin{array}{l}96 \% \text { and } 84 \% \text { retention of } j \text { in } \\
\text { current-time chronoamperometry } \\
\text { through } 10800 \mathrm{~s} \text { in alkaline and } \\
\text { acidic solution, respectively. }\end{array}$ & 21 \\
\hline GNPCSs-800 & $\begin{array}{l}\text { ZIF-8 } \\
\text { GO } \\
\text { PVP }\end{array}$ & 911 & $\begin{array}{l}\text { Graphene-based nitrogen- } \\
\text { doped porous carbon } \\
\text { sheets. }\end{array}$ & $0.1 \mathrm{M} \mathrm{KOH}$ & 0.957 & 0.857 & N/A & 3.98 & $\begin{array}{l}94 \% \text { retention of } j \text { in current-time } \\
\text { chronoamperometry through } 28000 \\
\text { s in alkaline solution. }\end{array}$ & 22 \\
\hline C-CZ-4-1000 & MWCNTs & 709.9 & $\begin{array}{l}\text { Carbon structure attached } \\
\text { on the CNT surface. }\end{array}$ & $0.1 \mathrm{M} \mathrm{KOH}$ & 1.03 & 0.887 & 49 & 3.99 & $\begin{array}{l}\text { No significant negative shift (half } \\
\text { wave) in ADT through } 5000 \text { cycles } \\
\text { in alkaline solution. }\end{array}$ & 23 \\
\hline $\begin{array}{l}\text { Co@NSC/MW } \\
\text { CNTs }\end{array}$ & $\begin{array}{c}(4-\text { py-TTF-4- } \\
\text { py })_{2} \mathrm{M}_{2}(\mathrm{PTA})_{4}(\mathrm{M} \\
\left.=\mathrm{Co}^{2+}\right) \\
\text { MWCNT }\end{array}$ & N/A & $\begin{array}{l}\text { Co nanoparticles } \\
\text { embedded in N, S co- } \\
\text { doped carbon layer and } \\
\text { supported on multi-walled } \\
\text { carbon nanotubes. }\end{array}$ & $0.1 \mathrm{M} \mathrm{KOH}$ & 0.843 & 0.782 & 49 & 3.9 & $\begin{array}{l}96.5 \% \text { retention of } j \text { in current-time } \\
\text { chronoamperometry through } 10000 \\
\mathrm{~s} \text { in alkaline solution. }\end{array}$ & 24 \\
\hline
\end{tabular}




\begin{tabular}{|c|c|c|c|c|c|c|c|c|c|c|}
\hline \multirow[t]{2}{*}{ Co-Zn-2 } & $\begin{array}{c}\text { ZIF-8 } \\
\text { ZIF-67 } \\
\text { Bimetallic(Zn/Co) }\end{array}$ & 429 & $\begin{array}{l}\text { 2D nanoplatelet with } \\
\text { honeycomb-like } \\
\text { nanostructure contains } \\
\text { metallic Co nanoparticles. }\end{array}$ & $0.1 \mathrm{M} \mathrm{KOH}$ & 0.976 & 0.849 & 57 & 3.96 & \multirow[t]{2}{*}{$\begin{array}{l}\sim 99.5 \% \text { retention of } j \text { in current-time } \\
\text { chronoamperometry through } 20000 \\
\mathrm{~s} \text { in alkaline solution. }\end{array}$} & \multirow[t]{2}{*}{25} \\
\hline & CoAl-LDH & & & $0.1 \mathrm{M} \mathrm{HClO}_{4}$ & 0.892 & 0.679 & N/A & $\sim 3.9$ & & \\
\hline \multirow[t]{2}{*}{ Coo.05,N,_pCNT } & $\begin{array}{l}\text { ZIF-8 } \\
\text { ZIF-67 }\end{array}$ & \multirow[t]{2}{*}{1125} & \multirow[t]{2}{*}{$\begin{array}{l}1 \mathrm{D} \text { Co and N-doped } \\
\text { hierarchically porous } \\
\text { carbon nanotubes. }\end{array}$} & \multirow[t]{2}{*}{$0.1 \mathrm{M} \mathrm{KOH}$} & \multirow[t]{2}{*}{1.00} & \multirow[t]{2}{*}{0.87} & \multirow[t]{2}{*}{ N/A } & \multirow[t]{2}{*}{$\sim 4.0$} & $\begin{array}{l}97 \% \text { retention of } j \text { in current-time } \\
\text { chronoamperometry through } 86400 \\
\mathrm{~s} \text { in alkaline solution. }\end{array}$ & \multirow[t]{2}{*}{26} \\
\hline & $\begin{array}{l}\text { Bimetallic }(\mathrm{Zn} / \mathrm{Co}) \\
\text { Porous tellurium } \\
\text { nanotubes }\end{array}$ & & & & & & & & $\begin{array}{l}\text { No significant negative shift (half } \\
\text { wave) in ADT through } 5000 \text { cycles } \\
\text { in alkaline solution. }\end{array}$ & \\
\hline Co@C-NCNT & HNTs & 541 & $\begin{array}{l}\text { Nitrogen-doped carbon } \\
\text { nanotubes with open end } \\
\text { and large inner cavity, } \\
\text { thin wall moderate } \\
\text { graphitization, and } \\
\text { decoration with carbon- } \\
\text { coated Co nanoparticles. }\end{array}$ & $0.1 \mathrm{M} \mathrm{HClO}_{4}$ & 0.795 & 0.691 & N/A & $\sim 4.0$ & $\begin{array}{l}84 \% \text { and } 83.8 \% \text { retention of } j \text { in } \\
\text { current-time chronoamperometry } \\
\text { through } 22000 \mathrm{~s} \text { in alkaline and } \\
\text { acidic solution, respectively. }\end{array}$ & 27 \\
\hline
\end{tabular}




\section{References}

(1) Hu, Z.; Guo, Z.; Zhang, Z.; Dou, M.; Wang, F. Bimetal zeolitic imidazolite framework-derived iron-, cobalt-and nitrogen-codoped carbon nanopolyhedra electrocatalyst for efficient oxygen reduction. ACS Appl. Mater. Interfaces 2018, 10 (15), 12651-12658.

(2) Guo, Z.; Zhang, Z.; Li, Z.; Dou, M.; Wang, F. Well-defined gradient Fe/Zn bimetal organic framework cylinders derived highly efficient iron-and nitrogen-codoped hierarchically porous carbon electrocatalysts towards oxygen reduction. Nano Energy 2019, 57, 108-117.

(3) Liu, T.; Zhao, P.; Hua, X.; Luo, W.; Chen, S.; Cheng, G. An Fe-N-C hybrid electrocatalyst derived from a bimetal-organic framework for efficient oxygen reduction. J. Mater. Chem. A 2016, 4 (29), 1135711364.

(4) Wang, X.; Zhang, H.; Lin, H.; Gupta, S.; Wang, C.; Tao, Z.; Fu, H.; Wang, T.; Zheng, J.; Wu, G. Directly converting Fe-doped metal-organic frameworks into highly active and stable Fe-NC catalysts for oxygen reduction in acid. Nano Energy 2016, 25, 110-119.

(5) Lai, Q.; Zheng, L.; Liang, Y.; He, J.; Zhao, J.; Chen, J. Metal-organic-framework-derived Fe-N/C electrocatalyst with five-coordinated $\mathrm{Fe}-\mathrm{N}_{\mathrm{x}}$ sites for advanced oxygen reduction in acid media. ACS Catal. 2017, 7 (3), 1655-1663.

(6) Deng, Y.; Dong, Y.; Wang, G.; Sun, K.; Shi, X.; Zheng, L.; Li, X.; Liao, S. Well-defined ZIF-derived $\mathrm{Fe}-\mathrm{N}$ codoped carbon nanoframes as efficient oxygen reduction catalysts. ACS Appl. Mater. Interfaces 2017, 9 (11), 9699-9709.

(7) Li, J.; Ghoshal, S.; Liang, W.; Sougrati, M.-T.; Jaouen, F.; Halevi, B.; McKinney, S.; McCool, G.; Ma, C.; Yuan, X. Structural and mechanistic basis for the high activity of $\mathrm{Fe}-\mathrm{N}-\mathrm{C}$ catalysts toward oxygen reduction. Energy Environ. Sci. 2016, 9 (7), 2418-2432.

(8) Strickland, K.; Miner, E.; Jia, Q.; Tylus, U.; Ramaswamy, N.; Liang, W.; Sougrati, M.-T.; Jaouen, F.; Mukerjee, S. Highly active oxygen reduction non-platinum group metal electrocatalyst without direct metal-nitrogen coordination. Nat. Commun. 2015, 6, 7343.

(9) Zhu, Q.-L.; Xia, W.; Zheng, L.-R.; Zou, R.; Liu, Z.; Xu, Q. Atomically dispersed Fe/N-doped hierarchical carbon architectures derived from a metal-organic framework composite for extremely efficient electrocatalysis. ACS Energy Lett. 2017, 2 (2), 504-511.

(10) Lastovina, T. A.; Budnyk, A. P.; Pimonova, Y. A.; Bugaev, A. L.; Fedorenko, A. G.; Dmitriev, V. P. Step-by-step synthesis of a heteroatom-doped carbon-based electrocatalyst for the oxygen reduction reaction. Electrochem. Commun 2018, 88, 83-87.

(11) Aijaz, A.; Masa, J.; Rösler, C.; Antoni, H.; Fischer, R. A.; Schuhmann, W.; Muhler, M. MOFTemplated Assembly Approach for Fe3C Nanoparticles Encapsulated in Bamboo-Like N-Doped CNTs: Highly Efficient Oxygen Reduction under Acidic and Basic Conditions. Chem. Eur. J. 2017, 23 (50), 12125-12130.

(12) Zhong, R.; Wu, Y.; Liang, Z.; Guo, W.; Zhi, C.; Qu, C.; Gao, S.; Zhu, B.; Zhang, H.; Zou, R. Fabricating hierarchically porous and $\mathrm{Fe}_{3} \mathrm{C}$-embeded nitrogen-rich carbon nanofibers as exceptional electocatalysts for oxygen reduction. Carbon 2019, 142, 115-122.

(13) Guan, B. Y.; Yu, L.; Lou, X. W. D. A dual-metal-organic-framework derived electrocatalyst for oxygen reduction. Energy Environ. Sci. 2016, 9 (10), 3092-3096.

(14) Qian, Y.; Cavanaugh, J.; Khan, I. A.; Wang, X.; Peng, Y.; Hu, Z.; Wang, Y.; Zhao, D. Fe/Fe ${ }_{3} \mathrm{C} / \mathrm{N}-$ Doped Carbon Materials from Metal-Organic Framework Composites as Highly Efficient Oxygen Reduction Reaction Electrocatalysts. ChemPlusChem 2016, 81 (8), 718-723.

(15) Yang, L.; Bai, Y.; Zhang, H.; Geng, J.; Shao, Z.; Yi, B. Nitrogen-doped porous carbon derived from Fe-MIL nanocrystals as an electrocatalyst for efficient oxygen reduction. $R S C A d v . \mathbf{2 0 1 7}, 7$ (36), 2261022618. 
(16) Wang, Y.; Chen, X.; Lin, Q.; Kong, A.; Zhai, Q.-G.; Xie, S.; Feng, P. Nanoporous carbon derived from a functionalized metal-organic framework as a highly efficient oxygen reduction electrocatalyst. Nanoscale 2017, 9 (2), 862-868.

(17) Zhao, P.; Hua, X.; Xu, W.; Luo, W.; Chen, S.; Cheng, G. Metal-organic framework-derived hybrid of $\mathrm{Fe}_{3} \mathrm{C}$ nanorod-encapsulated, N-doped CNTs on porous carbon sheets for highly efficient oxygen reduction and water oxidation. Catal. Sci. Technol. 2016, 6 (16), 6365-6371.

(18) Ye, Y.; Cai, F.; Yan, C.; Li, Y.; Wang, G.; Bao, X. Two-step pyrolysis of ZIF-8 functionalized with ammonium ferric citrate for efficient oxygen reduction reaction. J. Energy Chem. 2017, 26 (6), 1174-1180.

(19) Zhang, L.; Wang, X.; Wang, R.; Hong, M. Structural evolution from metal-organic framework to hybrids of nitrogen-doped porous carbon and carbon nanotubes for enhanced oxygen reduction activity. Chem. Mater. 2015, 27 (22), 7610-7618.

(20) Li, J.; Chen, Y.; Tang, Y.; Li, S.; Dong, H.; Li, K.; Han, M.; Lan, Y.-Q.; Bao, J.; Dai, Z. Metal-organic framework templated nitrogen and sulfur co-doped porous carbons as highly efficient metal-free electrocatalysts for oxygen reduction reactions. J. Mater. Chem. A 2014, 2 (18), 6316-6319.

(21) Wei, J.; Hu, Y.; Liang, Y.; Kong, B.; Zhang, J.; Song, J.; Bao, Q.; Simon, G. P.; Jiang, S. P.; Wang, H. Nitrogen-doped nanoporous carbon/graphene nano-sandwiches: Synthesis and application for efficient oxygen reduction. Adv. Funct. Mater. 2015, 25 (36), 5768-5777.

(22) Zhong, H. x.; Wang, J.; Zhang, Y. w.; Xu, W. 1.; Xing, W.; Xu, D.; Zhang, Y. f.; Zhang, X. b. ZIF-8 derived graphene-based nitrogen-doped porous carbon sheets as highly efficient and durable oxygen reduction electrocatalysts. Angew. Chem. Int. Ed. 2014, 53 (51), 14235-14239.

(23) Ge, L.; Yang, Y.; Wang, L.; Zhou, W.; De Marco, R.; Chen, Z.; Zou, J.; Zhu, Z. High activity electrocatalysts from metal-organic framework-carbon nanotube templates for the oxygen reduction reaction. Carbon 2015, 82, 417-424.

(24) Li, S.; Jiang, Z.; Xiao, X.; Chen, W.; Tian, X.; Hao, X.; Jiang, Z.-J. MOF-derived Co nanoparticles embedded in N, S-codoped carbon layer/MWCNTs for efficient oxygen reduction in alkaline media. Ionics 2019, 25 (2), 785-796.

(25) Li, Z.; Shao, M.; Zhou, L.; Yang, Q.; Zhang, C.; Wei, M.; Evans, D. G.; Duan, X. Carbon-based electrocatalyst derived from bimetallic metal-organic framework arrays for high performance oxygen reduction. Nano Energy 2016, 25, 100-109.

(26) Ahn, S. H.; Klein, M. J.; Manthiram, A. 1D Co-and N-Doped Hierarchically Porous Carbon Nanotubes Derived from Bimetallic Metal Organic Framework for Efficient Oxygen and Tri-iodide Reduction Reactions. Adv. Energy Mater. 2017, 7 (7), 1601979.

(27) Li, R.; Wang, X.; Dong, Y.; Pan, X.; Liu, X.; Zhao, Z.; Qiu, J. Nitrogen-doped carbon nanotubes decorated with cobalt nanoparticles derived from zeolitic imidazolate framework-67 for highly efficient oxygen reduction reaction electrocatalysis. Carbon 2018, 132, 580-588. 